\title{
Progressive delamination analysis through two-way global-local coupling approach preserving energy dissipation for single-mode and mixed-mode loading
}

\author{
Margarita Akterskaia ${ }^{\mathrm{a}, *}$, Pedro P. Camanho ${ }^{\mathrm{b}}$, Eelco Jansen ${ }^{\mathrm{a}}$, Albertino \\ Arteiro $^{\mathrm{b}}$, Raimund Rolfes ${ }^{\mathrm{a}}$ \\ ${ }^{a}$ Institute of Structural Analysis, Leibniz Universität Hannover, Appelstr. 9A, 30167 \\ Hannover, Germany \\ ${ }^{b}$ DEMec, Faculdade de Engenharia, Universidade do Porto, Rua Dr. Roberto Frias, \\ 4200-465 Porto, Portugal
}

\begin{abstract}
Together with fiber breakage and matrix cracking, delamination is one of the common damage mechanisms occurring in laminated fiber-reinforced composite structures. Delamination initiates due to the relatively low interlaminar strength of adjacent plies. Delamination onset and propagation can be induced by various combinations of loads and usually leads to a significant reduction of the load-carrying capacity of the structure. For this reason, an efficient and reliable progressive failure analysis capability is required. In this work, the delamination process is simulated by means of a two-way global-local coupling approach. In particular, within this novel global-local approach a method is introduced that ensures the preservation of the dissipated energy when switching between the global and local level. This approach is tested and illustrated under single-mode I and II, and mixed-mode loading in the double cantilever beam (DCB), the end-notched flexure (ENF) and the mixed-mode bending (MMB) benchmark tests, respectively, and the results are compared to available analytical solutions. Finally, the developed method has been applied to a one-stringer stiffened panel and a good agreement was attained compared to the full reference solution.
\end{abstract}

\footnotetext{
* Corresponding author

Email address: m.akterskaia@isd.uni-hannover.de (Margarita Akterskaia)
} 
Keywords: Composite structures, DCB tests, ENF tests, MMB tests, Progressive failure analysis, Multiscale analysis, Global-local method, Skin-stringer debonding, Delamination

\section{Introduction}

Composite laminated stiffened panels are widely used in aircraft design as components of fuselages because of their excellent material properties, such as high strength and stiffness to weight ratio. To increase the ultimate load of 5 these structures and to exploit possible reserves, reliable simulations of postbuckling behavior of thin-walled structures are required [1]. For this reason, an accurate prediction of the failure behavior of composite structures is of great importance. One of the most common methods is to employ a material degradation model to perform a realistic failure analysis and to accurately detect the final collapse. Due to high computational costs that numerical simulation of the full structure requires, global-local approaches have been developed as a reliable and efficient tool to study localized nonlinearities, such as onset and evolution of damage, for example. A two-way global-local coupling method to simulate the post-buckling progressive failure behavior of composite stiffened panels with intralaminar damage and skin-stringer separation was developed in earlier work [2, 3. In this method, first, critical areas are defined at the global level and local models with a considerably finer mesh are created by means of a submodeling technique. Secondly, a local model analysis is conducted. Cohesive elements are applied to model delamination with special attention to the exchange of information between the global and local models. The global-local coupling loops are repeated until final global failure occurs. In the earlier work [4, the averaged damage scalar parameter obtained from the cohesive elements of the local model was utilized to determine the degraded stiffness of the adhesive layer in the global model.

In the current study, a global-local method is formulated ensuring the preservation of dissipated energy between the global and local analysis for single-mode 
and mixed-mode loadings by a novel strategy of information transfer from the local to the global level. To achieve this, the degraded stiffness of the adhesive layer in the global model is calculated based on the energy dissipated due to delamination in the local model. The dissipated energy in the local model is obtained for each area of cohesive elements that corresponds to each discrete element representing the adhesive layer in the global model. Subsequently, the degraded stiffness of each global adhesive element is calculated ensuring that the same amount of energy will be dissipated as in the cohesive elements of the local model. Its application to the double cantilever beam (DCB) and the mixed-mode bending (MMB) benchmark test, respectively, is demonstrated with a comparison to analytical solutions based on Fracture Mechanics. The skin-stringer debonding is investigated in a one-stringer stiffened panel with the developed global-local methodology and compared to the full 3D reference 40 solution.

\subsection{Continuum and discrete elements for delamination}

Delamination takes place in interface layers of composite laminates and can lead to drastic consequences, such as the reduction in load-bearing capacities of the full structures. Delamination onset and propagation occurs due to the relatively low interlaminar strength of adjacent plies and under various combinations of loads. There are two main modeling techniques to simulate delamination: the Virtual Crack Closure Technique (VCCT) [5] [6], and cohesive interface elements [7] 8] 9]. The VCCT, which is based on Fracture Mechanics, requires information about the place where the crack initiates. This is a disadvantage of this method when applying it to large and complex structures where delamination onset is usually not known a priori. Cohesive Zone Modeling $(\mathrm{CZM})$ [10] 11] 12] is based on the hypothesis that tractions keep together the softening region in front of the crack tip. In this work, a common bilinear traction-separation law is used which assumes that an initially linear behavior is followed by a softening region when the strength is attained. The fracture toughness $G_{c}$ is equal to the area under the traction-separation curve, refer to 
Fig. 1, and total crack opening takes place when this toughness is completely dissipated.

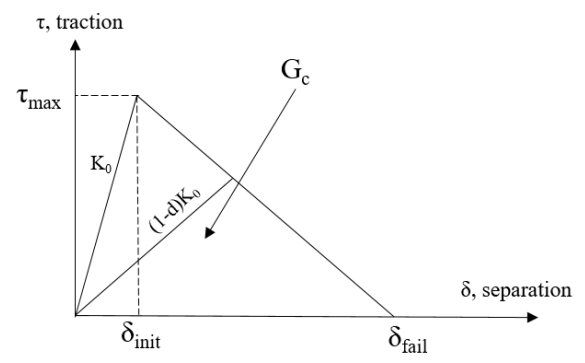

Figure 1: Bilinear traction-separation law.

On the one hand, the Cohesive Zone Model could be applied in continuum form with interface elements, and on the other hand, it could be implemented by using point-wise discrete elements in the crack zone. The concept of interface elements was extensively developed by many authors, e.g., Allix and Ladevèze [13, Camanho and Dávila [9] and Turon et al. [14]. The discrete cohesive zone approach was formulated in the works of Borg et al. 15, Wisnom and Chang [16], Xie and Waas [17, Hallett and Wisnom [18, and Jiang et al. 19]. Liu et al. 20] implemented discrete two-dimensional spring-like elements with a softening behavior to simulate delamination and fiber debonding and matrix cracking. This work was extended by Jimenez et al. [21] for the mixed-mode delamination fatigue analysis. Later studies were also conducted by Cabello et al. 22] in the application to a DCB bonded joint in an analytical solution. Both continuum and discrete modeling approaches require a relatively high number of elements to accurately estimate tractions in interface elements. For this reason, global-local methods are used to reduce computational time and to provide an accurate solution in damaged areas.

\subsection{Global-local methods for delamination modeling}

Global-local methods are paramount for simulating progressive damage in large and complex structures due to their effectiveness in reducing computational efforts without compromising the accuracy of damage analysis, which 
would happen if using a coarse analysis. Delamination is one of the critical failure modes that can significantly reduce the load-carrying capacity of a structure and lead to the final collapse. The current research aims at investigating delamination between adjacent layers of laminated composites. An overview of existing global-local approaches for delamination analysis was given in [4]. These methods were applied to skin-stringer debonding in stiffened panels with different stringer shapes. It is useful to distinguish between one-way and twoway global-local coupling methods. The one-way coupling technique is based on the information transfer in one direction, either from the global to the local model, or less commonly, from the local to the global model. Contrarily, two-way coupling incorporates information exchange between global and local models in both directions. Orifici et al. 23] calculated global and local models separately. Delamination onset was predicted at the local level, while at the global level, delamination evolution was modeled using VCCT. Reinoso et al. 24] applied the one-way coupling method, comparing the submodeling procedure and the shell-to-solid coupling technique for the local model. Delamination was simulated using cohesive elements at the local level. Vescovini et al. [25] conducted one-way coupling using global and local models composed of shell elements. Cohesive elements were used at the local level. Borrelli et al. [26] examined delamination growth with global and local models calculated simultaneously, as the local model represented a refined part of the global model around the delaminated area. The global model consisted of shell elements, whereas solid elements were used for the local model. Delamination was modeled with the modified virtual crack closure technique (MVCCT). Bettinotti et al. 27. conducted a numerical analysis for a composite panel under high-velocity impact. A local model was incorporated into the global model. Continuum shell elements were used for both models and cohesive elements were applied to the local model. Saavedra et al. 28] utilized the Domain Decomposition Method (DDM) to perform a multiscale analysis where delamination was modeled with cohesive interfaces. The influence of boundary conditions and the geometry of the model on the convergence rate was demonstrated. 


\subsection{Objectives}

A global-local two-way coupling method for modeling the initiation and further propagation of skin-stringer separation in stiffened panels has been developed earlier [4], 29]. The method was validated by a comparison to experimental results of a one-stringer panel with and without initial skin-stringer debonding. tively coarse mesh. Discrete elements were used to represent the interface layer. The local models were created based on the areas where the debonding was predicted at the global level. Solid elements were utilized in local models for the skin and the stringer with finer mesh. The interface layer of the local model was modeled with cohesive elements. Information exchange from the local to the global level was performed via transfer of the degraded interface stiffness of each particular global interface element. The global stiffness was degraded following the averaging of scalar damage variables obtained from the local model. Although this approach has demonstrated excellent results, it did not ensure 125 the preservation of energy dissipation across both levels.

The objective of this work is to develop a new global-local coupling methodology that will allow a shell type model with a coarse mesh in combination with local models with a fine solid mesh and cohesive elements to simulate the delamination behavior of a full reference model. A local-global information transfer is based on the energy dissipation at both global and local levels. Single-mode and mixed-mode loadings are considered. The approach is tested and verified under single-mode loading test cases, such as the double cantilever beam (DCB) test and the end notched flexure (ENF) test for the modes I and II respectively. Mixed-mode loading was verified with the mixed-mode bending (MMB) test

[30]. These tests are standardized by ASTM. All global-local coupling results have been compared to reference solutions obtained with fine discretization and to available analytical solutions. Finally, the proposed approach has been applied to a single-stiffened panel and the results obtained were compared to a reference full 3D solution. 


\section{Analysis Methodology}

To the authors' knowledge, previous studies addressing global-local delamination analysis have not been applied to standard test cases for validation on simpler, single- and mixed-mode loading cases. In this section, global-local analysis of single-mode and mixed-mode delamination was performed to drive the formulation and validate the global-local method proposed herein (see also Section 3). Further validation at a more complex level will be presented in Section 4.

\subsection{Global-local approach}

The global-local method consists of several coupling loops repeated consecutively. First, the global model is created using a coarse mesh. Shell elements are utilized due to to their advantage in terms of low computational time and their ability to accurately predict structural behavior of slender structures.

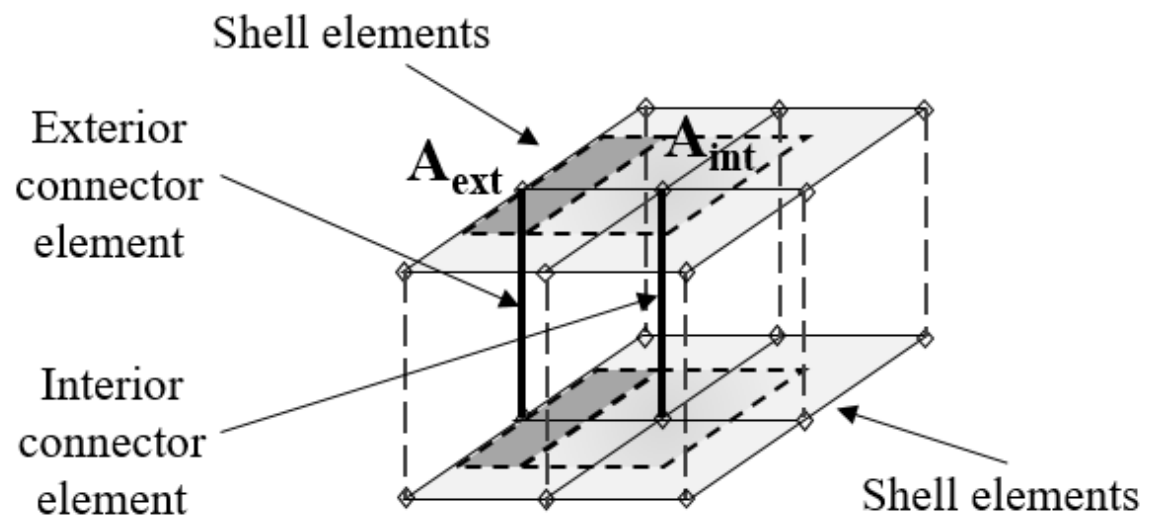

Figure 2: Shell elements connected by connector elements.

Discrete elements of the connector type in Abaqus tie the corresponding nodes of shell elements as shown in Fig. 2. These elements enable the definition of stiffness in three directions. Following the stiffness definition of connector elements, the normal and shear stiffness are calculated as:

$$
K_{n}=\frac{E A}{t}
$$




$$
K_{s}=\frac{G A}{t}
$$

where $E$ and $G$ are the Young's modulus and shear modulus of the adhesive zone, respectively, $A$ is the area associated with a node and $t$ is the interface thickness. The critical areas where debonding might take place are determined by the quadratic stress criterion commonly used for cohesive elements:

$$
\left(\frac{<\sigma_{n}>}{\tau_{I}}\right)^{2}+\left(\frac{\sigma_{s}}{\tau_{I I}}\right)^{2}+\left(\frac{\sigma_{t}}{\tau_{I I I}}\right)^{2}=1
$$

Here $\langle\ldots\rangle$ is used for the Macauley brackets operator in order to exclude compression from interface separation. $\sigma_{n}$ is the stress acting in the normal through-thickness direction, $\sigma_{s}$ and $\sigma_{t}$ are shear stresses, and $\tau_{I}, \tau_{I I}, \tau_{I I I}$ are the corresponding strengths.

Normal and shear stresses at the nodes of connector elements are calculated from forces in connector elements distinguishing between free edge and internal nodal areas that are tied by connector elements:

$$
\sigma_{i 3}=\frac{F_{i}}{A_{e l}}, \quad i=1,2,3
$$

where $F_{i}$ is a nodal force, and $A_{e l}$ determines a nodal area of applied force and taken as the sum of one quarter of each element area tied to that node. Therefore, $A_{e l}$ either represents the full in-plane area of the shell element $A_{\text {int }}$, referring to Fig. 2 for interior connectors, or half of this area denoted as $A_{\text {ext }}$ corresponding to the case when connectors tie the edges, or a quarter if connectors tie corner elements. Index $i$ specifies local Cartesian directions. $\sigma_{33}$ corresponds to the normal stress that acts through the thickness, $\sigma_{13}$ and $\sigma_{23}$ are two in-plane shear stresses. In Eq. 1, the penalty stiffness definition includes non-material parameters, such as nodal area $A$ and thickness $t$. The force $F_{i}$ from Eq. 4 is proportional to the corresponding stiffness which means that the stresses $\sigma_{i 3}$ are independent from the nodal area and depend only on the thickness of the adhesive layer. Connector elements that tie four-node 2D shell elements are shown in Fig. 2, indicating the nodal areas $A_{\text {ext }}$ and $A_{\text {int }}$. 
Based on the areas detected as probable damaged regions, the local models are created with a finer mesh and solid elements to capture full 3D stress states. The interface layer is modeled with cohesive elements with a bilinear tractionseparation law shown in Fig. 1 to simulate delamination. Displacements as kinematic boundary conditions are transferred to the boundaries from the global to the local model through a submodeling procedure. Moreover, the stress-based criterion, see Eq. 3, is used to predict the initiation of delamination, whereas the Benzeggagh and Kenane criterion 31 is applied for modeling the delamination propagation. This criterion was developed for mixed-mode loading and is used later for this case, see Eq. 15, but it is also applicable to single-mode loading. A scalar damage variable $d$ varies from 0 , when the crack is not yet opened and no energy is dissipated, until 1, when the crack is fully opened and energy is completely dissipated. The cohesive element stiffness is represented as follows [9]:

$$
K= \begin{cases}K_{0} & \delta<\delta_{\text {init }} \\ (1-d) K_{0} & \delta_{\text {init }}<\delta<\delta_{\text {fail }} \\ 0 & \delta>\delta_{\text {fail }}\end{cases}
$$

where $K_{0}$ is the initial penalty stiffness, the displacement $\delta$ is changed from 0 to $\delta_{\text {init }}$, which corresponds to crack initiation, to $\delta_{\text {fail }}$, which is the full crack opening displacement. The initial stiffness of the cohesive element is defined similarly to the global model interface stiffness:

$$
K_{\text {local }_{n}}=\frac{E}{t}
$$

200

$$
K_{\text {local }_{s}}=\frac{G}{t}
$$

where $K_{\text {local }_{n}}$ and $K_{\text {local }_{s}}$ are cohesive normal and shear stiffnesses, respectively.

For reasons of simplicity, a global-local analysis developed for progressive failure analysis in application to stiffened panels employed an averaged scalar variable $d$ to transfer damage information from the local to the global level 4. This method, based on the averaging $d$-parameter, demonstrated good 
results. However, in order to preserve energy dissipated at the local level due to delamination evolution and to degrade global interface stiffness accordingly, the following procedure is used. First, similarly to the previous approach, a mapping procedure is necessary to allocate corresponding cohesive elements to one global connector element. Energy dissipated by these cohesive elements should be equal to the energy dissipated due to stiffness degradation by one connector element.

$$
E_{\text {diss.local }}=\sum_{i=1}^{N} E_{\text {diss.local }, i}=E_{\text {diss.global }}
$$

where $E_{\text {diss.local, } i}$ is the dissipated energy of a cohesive element, $i$ denotes one of $N$ local cohesive elements from the region corresponding to one connector element, and $E_{\text {diss.global }}$ is the dissipated energy of one connector element due to delamination. A developed method allowing the calculation of energies dissipated at the global level based on the information from the local level is discussed in detail in the following sections for single-mode and mixed-mode loadings.

When the global degraded stiffness is recalculated for each particular connector element, the global analysis is performed again to check if no critical areas appeared due to stress redistribution. Initial global interface stiffness is used until the increment when the damage was detected first. From that increment, global interface stiffness is updated to degraded. It is important to notice, that as a result of the mapping procedure correlating global interface elements to the region of local elements that usually consists of more than one element due to mesh refinement, all global interface elements might have different degraded stiffnesses. The applied load is increased until the moment when new damaged areas are found in the global model and the global-local coupling procedure is repeated until the final collapse occurs.

Hence, the coupling global-local loop includes three internal stages:

1. Global analysis to detect probable areas of delamination initiation and to determine the position and geometry of the local models.

2. Local analysis with cohesive elements to accurately detect the onset of delamination and to observe the delamination evolution. Energy dissipated 
from the later process is obtained.

3. The global interface stiffness is calculated based on the dissipated energy of cohesive elements and transferred back to the global level.

These coupling loops are repeated within one coupling step with the same load level until convergence in reaction forces is reached. The new coupling step and, hence, the increase of the displacement load applied at the global level is defined by one of the following conditions. Either new local damage is detected or the previous damaged area is extended with the increase of the load. Therefore, the location and extension of the local models is not defined $a$ priori and the method can be applied to generic cases. That means that local models can be created and updated based on the knowledge of the damage sizes at the regions identified after the global model analysis in a partially automated process. Using a Python script, the criterion for interlaminar damage is applied in all increments of the global model and interface elements in order to determine the increment when the new elements are damaged. Based on this information the displacement when the new elements satisfy the failure criterion becomes a displacement of the coupling step and the global-local procedure is started.

\subsection{Single-mode loading}

In order to obtain a degraded stiffness of each connector element for the single-mode loading, the following equations should be solved using known dissipated energy. The cohesive traction-separation curve and discrete forceseparation curves for a mode I loading are shown in Fig. 3.

First, the displacement of the final separation in the cohesive element is defined:

$$
\delta_{\max }=\frac{2 G_{I}}{\tau}
$$

Then for the force-separation curve of the connector element in the softening region with force $F^{*}$ and opening $\delta$ :

$$
F^{*}=\left(A-\frac{E_{\text {diss }}}{G_{I}}\right) \tau
$$



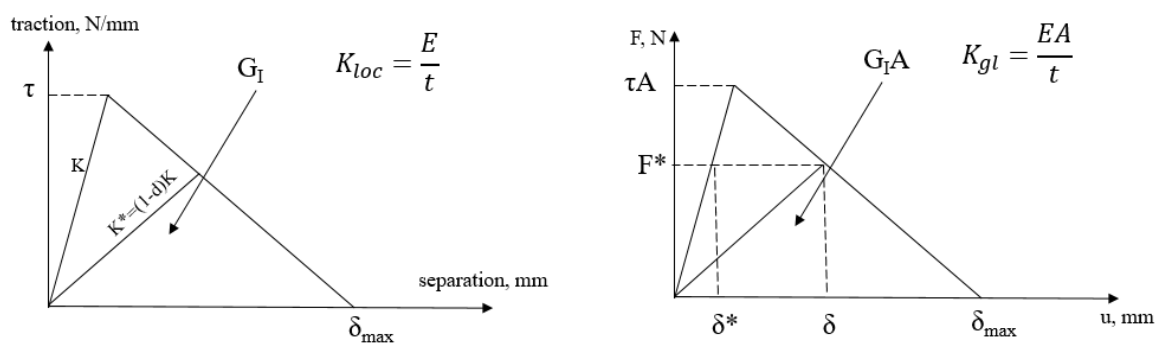

Figure 3: Traction-separation law for cohesive elements (left) and for connector elements (right).

The global dissipated energy can be obtained as follows:

$$
E_{\text {diss }}=\frac{1}{2}\left(\delta-\delta^{*}\right) \tau A
$$

From the same plot in Fig. 3 (right), $\delta^{*}$ is defined:

$$
\delta^{*}=\frac{F^{*}}{K_{g l}}
$$

Using Eq. 10, 11 and 12 ,

$$
\delta=\frac{2 E_{\text {diss }}}{\tau A}+\left(A-\frac{E_{\text {diss }}}{G_{I}}\right) \frac{\tau}{K_{g l}}
$$

Knowing $F^{*}$ and $\delta$, a new degraded global stiffness of a particular connector element could be defined as:

$$
K_{g l}^{*}=\frac{F^{*}}{\delta}
$$

Both normal and shear stiffness of the connector elements are updated based on previous equations and applied within the next step to the global model.

\subsection{Mixed-mode loading}

The mixed-mode fracture toughness is calculated using the Benzeggagh and

Kenane criterion 31 extended to the 3D case:

$$
G_{c}=G_{I c}+\left(G_{I I c}-G_{I c}\right)\left(\frac{G_{I I}+G_{I I I}}{G_{I}+G_{I I}+G_{I I I}}\right)^{\eta}
$$


where $G_{I c}$ and $G_{I I c}$ are the mode I and II fracture toughness and $G_{I}, G_{I I}, G_{I I I}$ are the single-mode energy release rates corresponding to the fracture modes I, II and III and their sum is the total energy release rate.

The value in brackets could be obtained directly from Abaqus for the delamination evolution and, hence, the mixed-mode energy release rate is assumed to be known. The maximum traction is defined as follows:

$$
\tau=\sqrt{\tau_{I}^{2}+\tau_{I I}^{2}+\tau_{I I I}^{2}}
$$

Following these assumptions, the degraded mixed-mode stiffness of the connector element can be calculated for the mixed-mode loading as:

$$
K_{\text {mixed }}=K_{I}+\left(K_{I I}-K_{I}\right)\left(\frac{G_{I I}+G_{I I I}}{G_{I}+G_{I I}+G_{I I I}}\right)^{\eta}
$$

which is also derived similarly by Turon et al. 32 .

280

\section{Validation and verification examples}

In this section, the global-local approach based on preservation of dissipated energies between global and local levels is applied to DCB, ENF and MMB test cases and compared to analytical solutions [33.

\subsection{Double cantilever beam (DCB) specimen}

285

The selected DCB specimen shown in Fig. 4 is a CFRP laminate with unidirectional fibers in longitudinal direction, which is $150 \mathrm{~mm}$ long, $20.0 \mathrm{~mm}$ wide, with arms of $1.55 \mathrm{~mm}$ thickness and an initial crack of $35 \mathrm{~mm}$. The geometry and material properties for this specimen are listed in Tables 1 and 2 , respectively. The interface properties used for cohesive elements in the local model are listed in Table 3

\subsubsection{Reference model}

The reference model was created composed of solid elements to model the arms and cohesive elements of $0.01 \mathrm{~mm}$ thickness, which is small enough compared to the maximum values [34. The influence of the viscosity parameter, 
Table 1: Geometry of the DCB specimen.

\begin{tabular}{ll}
\hline Description & Value \\
\hline Length, $L(\mathrm{~mm})$ & 150 \\
Width, $b(\mathrm{~mm})$ & 20 \\
Half of the specimen thickness, $h(\mathrm{~mm})$ & 1.55 \\
Initial crack length, $a_{0}(\mathrm{~mm})$ & 35 \\
\hline
\end{tabular}

Table 2: Material data for composite and adhesive.

\begin{tabular}{ll}
\hline Stiffness properties & Value \\
\hline Young's modulus in 1-direction, $E_{11}(\mathrm{GPa})$ & 171.4 \\
Young's modulus in 2-direction, $E_{22}(\mathrm{GPa})$ & 9.08 \\
Shear modulus in 12-plane, $G_{12}(\mathrm{GPa})$ & 5.29 \\
Shear modulus in 23-plane, $G_{23}(\mathrm{GPa})$ & 3.24 \\
Young's modulus for adhesive, $E_{a d h}(\mathrm{GPa})$ & 3.00 \\
Poisson's ratio, $\nu_{12}$ & 0.32 \\
Poisson's ratio, $\nu_{23}$ & 0.4 \\
Poisson's ratio for adhesive, $\nu_{a d h}$ & 0.4 \\
\hline
\end{tabular}

Table 3: Material data for interface.

\begin{tabular}{ll}
\hline Cohesive element properties & Value \\
\hline Interfacial strength, mode I, $\tau_{I}(\mathrm{MPa})$ & 30.0 \\
Interfacial strength, mode II and III, $\tau_{I I}, \tau_{I I I}(\mathrm{MPa})$ & 50.6 \\
Fracture toughness, mode I, $G_{I c}(\mathrm{~N} / \mathrm{mm})$ & 0.277 \\
Fracture toughness, mode II and III, $G_{I I c}, G_{I I I c}(\mathrm{~N} / \mathrm{mm})$ & 0.788 \\
$\eta$ & 1.634 \\
\hline
\end{tabular}




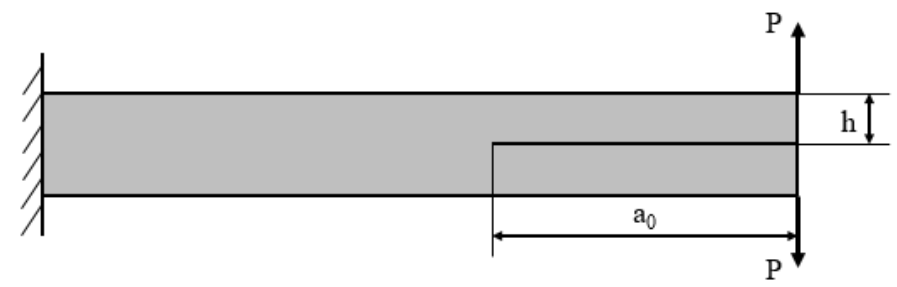

Figure 4: Double cantilever beam specimen.

295

the number of cohesive elements per solid element, the mesh size and the number of solid elements was investigated to select the suitable parameters for the local models assuming that their influence and importance are the same for the reference and local models.

\section{Effect of viscosity parameter}

Three analyses were conducted for the full reference model created with solid elements of $1 \mathrm{~mm}$ in-plane size, one cohesive element per solid element and 4 solid elements through the thickness of each arm, see Fig. 6. No viscosity, $10^{-5}$ and $10^{-7}$ viscosity parameters were chosen. Load-displacement curves for all three solutions are presented in Fig. 5 with comparison to the analytical solution. With no viscosity, the analysis failed to converge in reasonable time. The load-displacement curve of the analysis with a viscosity parameter of $10^{-5}$ demonstrates relatively high oscillations in the softening region compared to the analytical solution. An artificial viscosity parameter of $10^{-7}$ showed good agreement with the analytical curve and has been chosen as a good compromise.

\section{Effect of element number through the thickness}

The influence of the number of solid elements through the thickness was examined with one, two and four elements, see Fig. 6. The results of the loaddisplacement curves are presented in Fig. 7. It has been concluded that four elements through the thickness demonstrate results that are reasonably close to the analytical solution.

Effect of cohesive element number per solid element 


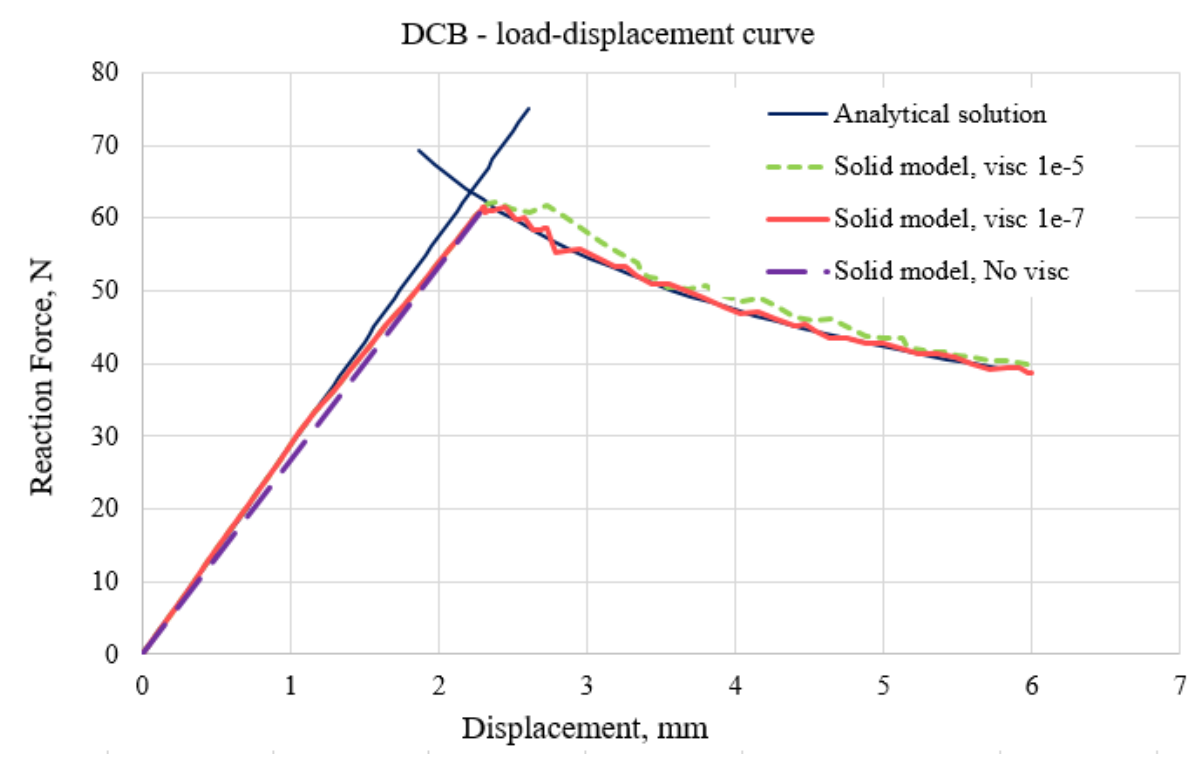

Figure 5: Load-displacement curve for the studies of viscosity parameter.

Further analyses were conducted with one and four cohesive elements per side of each solid element in the crack plane resulting in 1 and 16 cohesive elements connected to each solid element, respectively. Corresponding numerical meshes are demonstrated in Fig. 8. To overcome the difficulties related to non-corresponding nodes of solid and cohesive elements, it has been proposed to define element based surfaces and use a *TIE constraint to connect the surfaces. This approach has also been recommended by [24], though the author used second-order solid elements. The slave surface corresponded to cohesive elements and the master surface - to the solid elements. The results obtained for the DCB test case are presented in Fig. 9 compared to one cohesive element per solid element. The behavior of the model with four cohesive elements per solid element in the softening region could be explained by the problems related to the surface definition for the application of *TIE constraints when cohesive elements are deleted. Hence, it was concluded that for this type of structure and load, one cohesive element per solid element provides better results and should be chosen as a benchmark. 


\section{1 element through thickness}
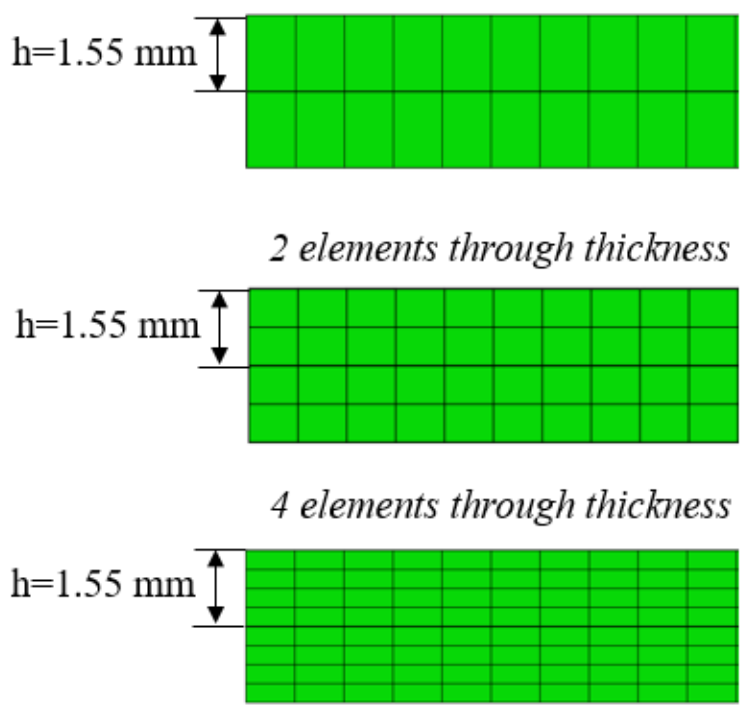

Figure 6: Through-thickness mesh densities with 1, 2 or 4 elements per arm.

Finally, the following parameters have been selected for the reference and local model: a viscosity of $10^{-7}$, four solid elements through the thickness and one cohesive element per solid element. Good agreement with analytical solution was attained for this reference model using the in-plane element size of $1 \mathrm{~mm}$. Reducing the element size would increase the computational cost without major gains in terms of accuracy, and increasing the element size is not desired, because solid elements are recommended to keep the aspect ratio close to one.

\subsubsection{Global model}

The global model was created with shell elements of $5 \mathrm{~mm}$ mesh size. Surface elements have been connected to the shell top and bottom surfaces using *TIE constraints connecting element-based surfaces, instead of using the offset parameter for shell elements. It allows a more accurate transfer of displacements to local models. The connector elements were used to tie the corresponding 


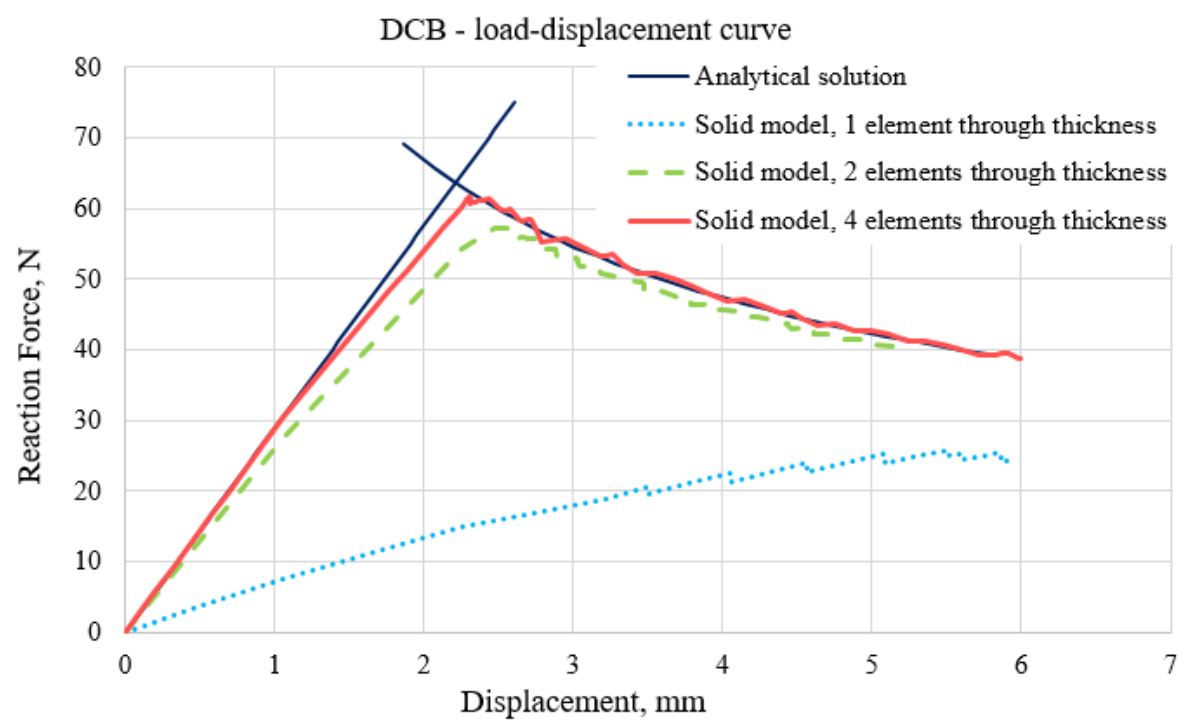

Figure 7: Load-displacement curve for the convergence studies of solid elements through thickness.

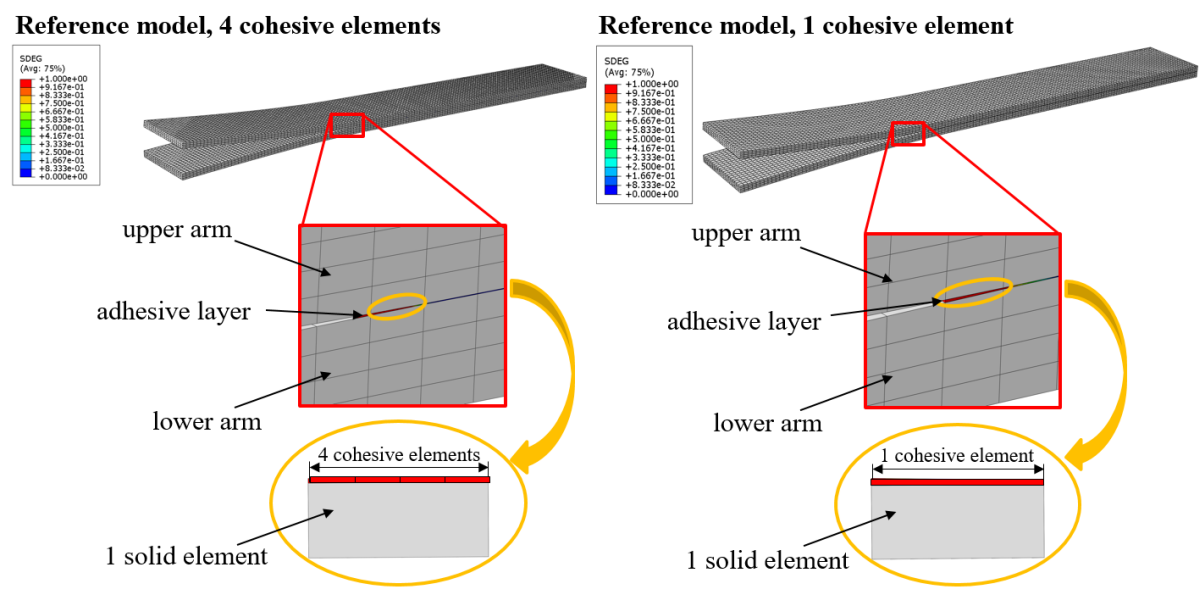

Figure 8: 3D reference models with four cohesive elements per one solid element in the crack plane (left) and one cohesive element per one solid element in the crack plane (right).

nodes of the upper and lower surfaces representing two arms. It allows the definition of actual forces and elongations in the connector elements. Using Eqs. 1 and 2, the normal and shear stiffnesses of cohesive elements were obtained 


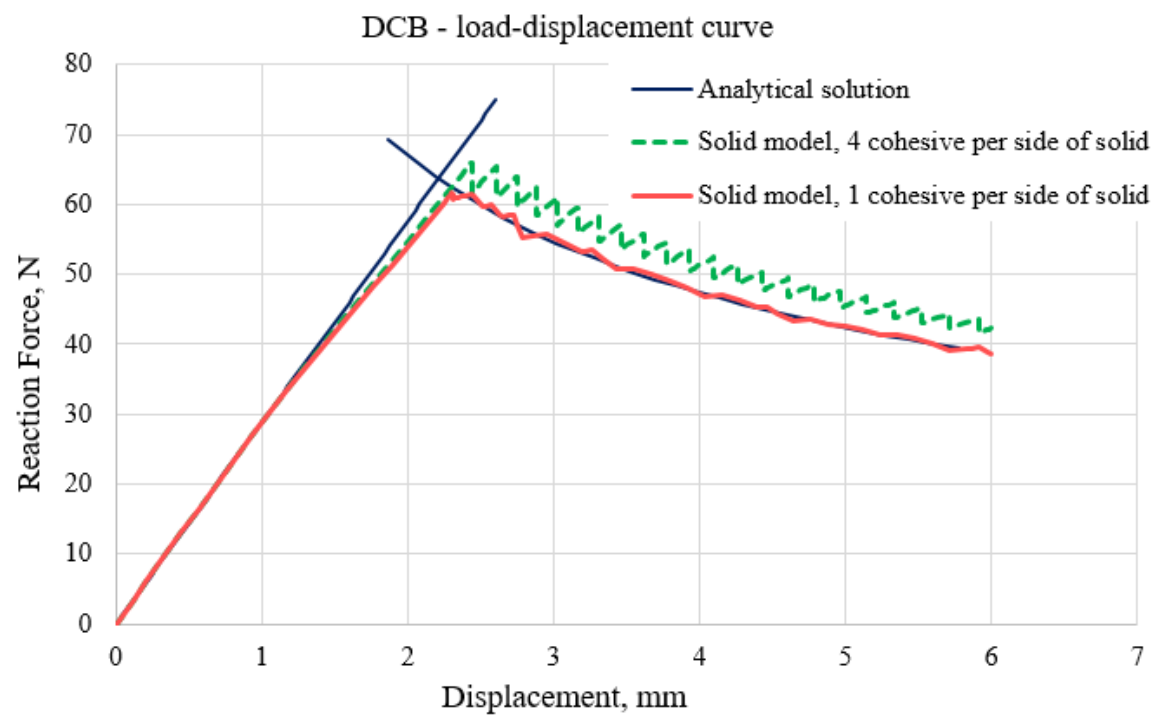

Figure 9: Load-displacement curve for the selection of the number of cohesive elements

as $7.5 \times 10^{6} \mathrm{~N} / \mathrm{mm}$ and $2.7 \times 10^{6} \mathrm{~N} / \mathrm{mm}$, respectively. The prediction of the delamination initiation at the global level utilizes the nodal area to calculate the stresses and the quadratic stress criterion from Eq. 3 .

\subsubsection{Local model}

The local model consisted of solid elements of $1 \mathrm{~mm}$ in-plane size and 4 elements through the thickness with one cohesive element per solid element corresponding to the reference solid model. A length of $40 \mathrm{~mm}$ for the local model with a pre-crack area of $15 \mathrm{~mm}$ has been chosen as short as possible and as long as required to allow the crack propagation, see Fig. 10.

\subsubsection{Results}

Five coupling steps have been performed with a consequent increase of applied displacement at the global level: $\mathrm{u}=2.5 \mathrm{~mm}, 3.0 \mathrm{~mm}, 4.0 \mathrm{~mm}, 5.0 \mathrm{~mm}$ and $6.0 \mathrm{~mm}$. That means that each arm was loaded with half of the displacement to reach the desired loading. The load-displacement curves of the analytical solution and coupling results are presented in Fig. 11. Several iterations were needed 


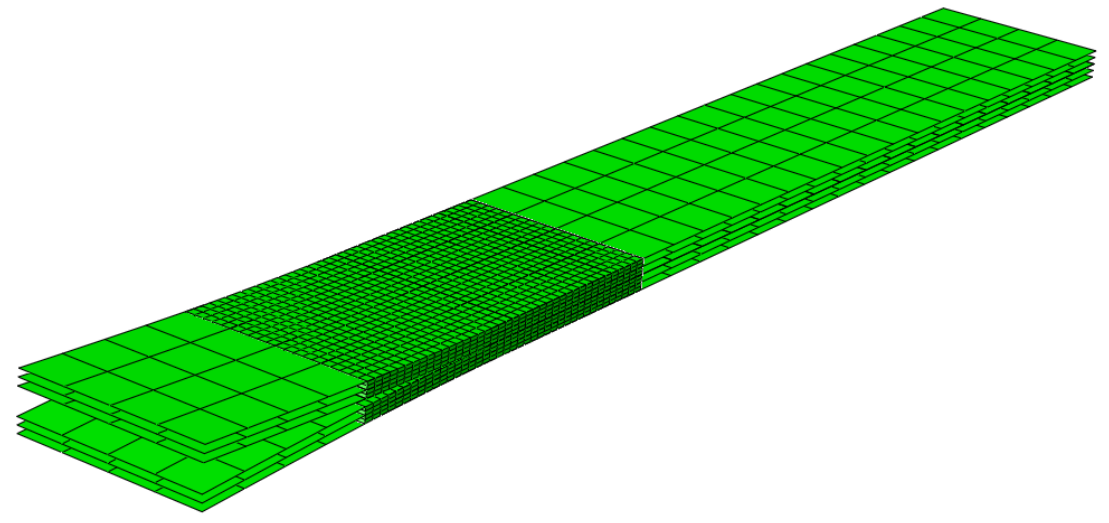

Figure 10: Overlay plot of global and local models for the DCB test.

to finish each coupling global-local step, which means that the applied load was increased only after a convergence in the resulting loads between global current and previous iteration was achieved. Each drop of the curve of the coupling results corresponds to the next global analysis where the degraded interface stiffness was used from the local analysis. Afterwards, as there is no other information, the global model continues to be linear, but it is updated again through the next coupling step results. This is why only the results that follow the drops in the global-local coupling step are assumed to be representative and comparable to the reference solution. Each local minimum value of each global coupling drop shows the force-displacement relation obtained with the globallocal approach and a good agreement with analytical and reference solutions is obtained.

In order to demonstrate convergence of the global-local approach to the analytical solution with an increasing number of coupling steps, a global-local analysis with eight coupling steps was performed. The arms of the DCB specimen were successively loaded with the applied displacement of $\mathrm{u}=2.5 \mathrm{~mm}, 2.7 \mathrm{~mm}$, $2.9 \mathrm{~mm}, 3.1 \mathrm{~mm}, 3.3 \mathrm{~mm}, 3.5 \mathrm{~mm}, 3.7 \mathrm{~mm}$ and $3.9 \mathrm{~mm}$. The results are shown 
in Fig. 12 and a good agreement between the global-local strategy and the analytical results is obtained in both linear and softening curves. The load drops of the global curve are explained by the sudden decrease of the material stiffness of some interface elements, which become smaller with increasing step number, 385 as expected. It should be noted here that an increase of the applied displacement from one coupling step to another at the global level is judged based on the damage evolution. But it should be recognized that the definition of the damage extension required to start a new global-local coupling step will depend on the problem and cannot be known a priori. In the present case, a finite number of coupling steps was defined upon damage initiation (5 in Fig. 11 and 8 in Fig. 12. In the first case (Fig. 11), damage growth was predicted at all displacement increments, prompting coupling steps that led to the load drops in Fig. 11. In the second case (Fig. 12, , some displacement increments led to negligible damage growth, as can be observed by the small load drops at $3.3 \mathrm{~mm}$ and $3.5 \mathrm{~mm}$ applied displacement. Hence, in general, the following recommendation applies: search for a compromise between an increase in the number of global-local coupling steps that leads to a better accuracy, but inevitably results in an undesired increase in computational time.

The energy dissipated during the cohesive elements damage was compared between the local and reference models for the case of the five coupling steps and is shown in Table 4. Both results correlate with each other. However, the highest difference in the initial global-local coupling step 1 is attributed to the fact that the displacements transferred from the global to the local models were higher than in the reference model at the same displacement level. The reason behind is that in the reference model the cohesive elements were damaged and deleted gradually within each load increment leading to the stress redistribution in the model, whereas in the global model the properties were not reduced until the first coupling step. It is also important to notice that the global model predicts the damage initiation slightly later as compared to the reference solution which ${ }_{410}$ is a consequence of a coarser mesh. That is a reason for the initial overestimation of the load and it is corrected through the global-local coupling step. 
Table 4: Energy dissipated in the local and reference models of the DCB specimen for 5 coupling steps.

\begin{tabular}{lcc}
\hline \multicolumn{3}{c}{ Dissipated energy, $\mathbf{N} \cdot \mathbf{m m}$} \\
\hline Coupling step & Local model & Reference model \\
\hline Coupling step 1, u=1.35 mm & 33.7 & 21.2 \\
Coupling step 2, $\mathrm{u}=1.50 \mathrm{~mm}$ & 42.6 & 34.9 \\
Coupling step 3, $\mathrm{u}=2.00 \mathrm{~mm}$ & 90.9 & 77.2 \\
Coupling step 4, $\mathrm{u}=2.50 \mathrm{~mm}$ & 117.0 & 105.7 \\
Coupling step 5, $\mathrm{u}=3.00 \mathrm{~mm}$ & 121.1 & 134.3 \\
\hline
\end{tabular}

Numerical analyses for global, local and reference models were performed under the same computational characteristics. Relative calculation times for these models for the analysis with five coupling steps are shown in Table 5. In order to obtain the numerical solution through the global-local method, global and local analyses should be carried out for each coupling step and each iteration that is required to achieve convergence in forces. Hence, the total global-local computational time is a sum of all these analyses times. It should be mentioned here that local models were recalculated from the previous step using the restart procedure in Abaqus which allowed for the considerable reduction of the computational effort. The relative calculation time for the global model changes from $10 \mathrm{~s}$ to $126 \mathrm{~s}$ for the first and last steps, respectively, whereas the full $3 \mathrm{D}$ reference model with cohesive elements required $3659 \mathrm{~s}$. The calculations of the local models lasted a maximum of $2473 \mathrm{~s}$ for the first local model and were lower for the local model restart procedure. However, the total computational time for the local models is $11457 \mathrm{~s}$ which is higher than for the reference model. This is due to the fact that, in this case, the local model is relatively large (40 mm length as compared to the reference model of $150 \mathrm{~mm}$ length). Both models have the same mesh densities, that implies comparable calculation costs, especially taking into account that following the global-local methodology, the 
Table 5: Computational characteristics of the models for the DCB test.

\begin{tabular}{lrrrr}
\hline Model & $\begin{array}{r}\text { Number of } \\
\text { nodes }\end{array}$ & $\begin{array}{r}\text { Number of } \\
\text { elements }\end{array}$ & $\begin{array}{r}\text { Degrees of } \\
\text { freedom }\end{array}$ & $\begin{array}{r}\text { Relative } \\
\text { computational } \\
\text { time, s }\end{array}$ \\
\hline Reference model & 101,014 & 58,516 & 206,394 & 3,659 \\
Global models & 1410 & 840 & 4,440 & 1,250 \\
Local models & 28,374 & 16,236 & 57,114 & 11,457 \\
\hline
\end{tabular}

local model should be numerically analysed several times. But it is important to note that the DCB test case has been chosen for the purpose of validating the approach developed for single-mode loading. In the case of localized damage with a relatively small local model, the global-local method based on the preservation of dissipated energies should be preferred due to the computational efficiency.

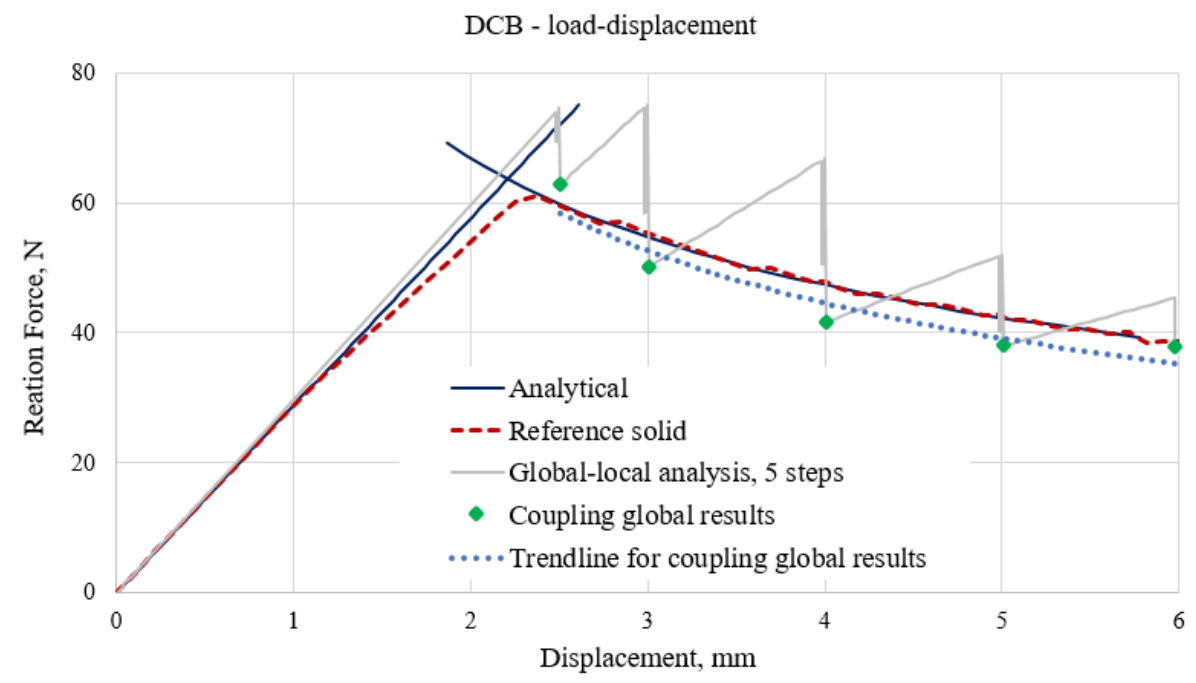

Figure 11: Load-displacement curves for the DCB test with 5 global-local coupling steps. 


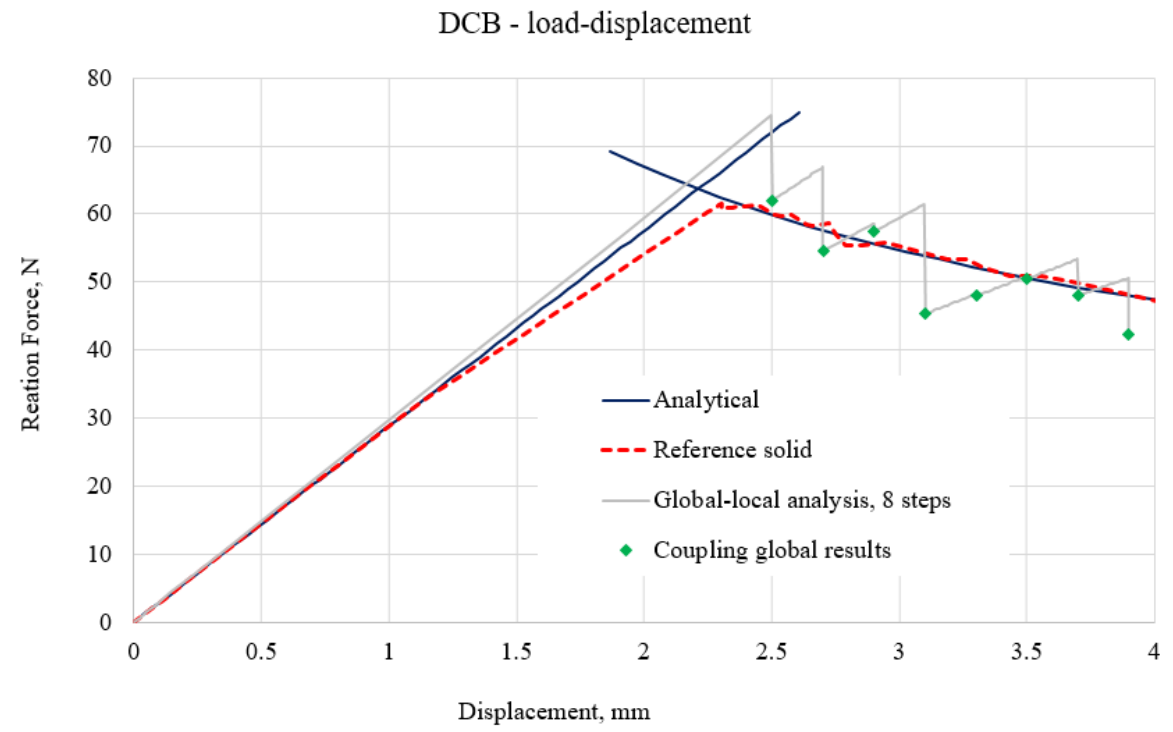

Figure 12: Load-displacement curves for the DCB test with 8 global-local coupling steps.

\subsection{End notched flexure (ENF) specimen}

The next test was conducted for mode II driven delamination for an end notched flexure (ENF) specimen, see Fig. 13. followed by comparison with the analytical solution. The material and cohesive element characteristics are the same as for the DCB test, see Tables 2 and 3, respectively. Geometry parameters are listed in Table 6

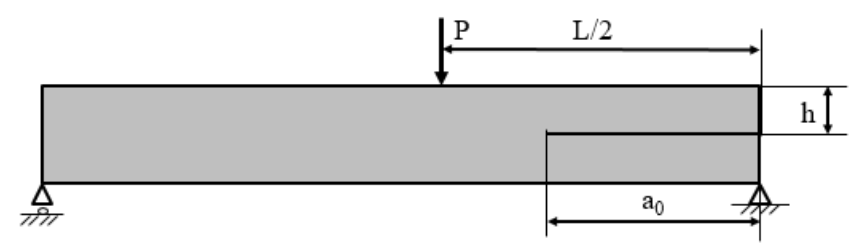

Figure 13: End notched flexure specimen.

The numerical parameters for the reference and local models were chosen as for the DCB specimen except for the viscosity parameter, as $10^{-5}$ has already 
Table 6: Geometry of the ENF specimen.

\begin{tabular}{ll}
\hline Description & Value \\
\hline Length, $L(\mathrm{~mm})$ & 100 \\
Distance of applied load, $L / 2(\mathrm{~mm})$ & 50 \\
Width, $b(\mathrm{~mm})$ & 20 \\
Half of the specimen thickness, $h(\mathrm{~mm})$ & 1.55 \\
Initial crack length, $a_{0}(\mathrm{~mm})$ & 35 \\
\hline
\end{tabular}

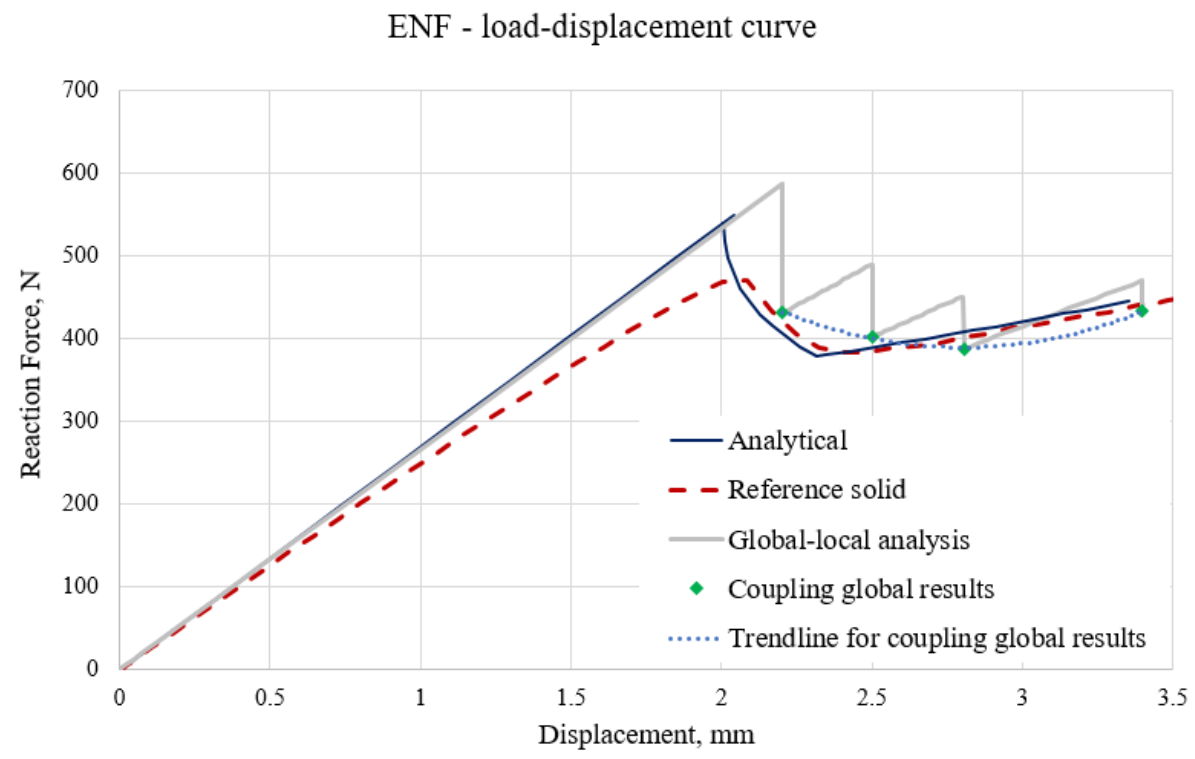

Figure 14: Load-displacement plot for the ENF specimen test.

The global-local analysis was carried out following the methodology suggested for the single-mode delamination. The size of the local model was the same as for the DCB test. A comparison of the load-displacement curves for the reference analysis, the analytical solution and the coupling procedure is demonstrated in Fig. 14. The global-local coupling analysis includes four consecutive 
Table 7: Geometry of the MMB specimen.

\begin{tabular}{ll}
\hline Description & Value \\
\hline Length, $L(\mathrm{~mm})$ & 150 \\
Distance of applied load, $c(\mathrm{~mm})$ & 63.18 \\
Width, $b(\mathrm{~mm})$ & 20 \\
Half of the specimen thickness, $h(\mathrm{~mm})$ & 1.55 \\
Initial crack length, $a_{0}(\mathrm{~mm})$ & 35 \\
\hline
\end{tabular}

steps based on prescribed displacements: $2.2 \mathrm{~mm}, 2.5 \mathrm{~mm}, 2.8 \mathrm{~mm}$ and $3.4 \mathrm{~mm}$. Good agreement between the analytical curve and the global-local simulation is achieved for the linear part and the second part of the softening curve. The difference in the first part of the nonlinear solution could be related to the fact that it is hard to predict the onset of the delamination with a coarse mesh at the global level. However, the application of the local analysis allowed to mitigate this with subsequent load increase.

\subsection{Mixed-mode bending (MMB) specimen}

A mixed-mode bending (MMB) specimen with $50 \%$ mode ratio has been selected to verify the global-local approach. The material properties are the same as for the DCB test, see Tables 2 and 3 and the geometry of the specimen is shown in Fig. 15 with the parameters given in Table 7.

The applied load was modeled by means of dummy nodes and their relation to the structure was ensured by specifying equations. The reference and local model parameters were chosen as previously with the viscosity parameter of $10^{-5}$. The load-displacement curves are presented in Fig. 16. Four coupling steps were used in the global-local analysis: increase of prescribed displacement to $6.0 \mathrm{~mm}, 6.5 \mathrm{~mm}, 7.0 \mathrm{~mm}$ and $7.5 \mathrm{~mm}$. Each global analysis was followed by local analysis simulations. The degraded global interface stiffnesses were 470 calculated for each global connector element and transferred back to the global 


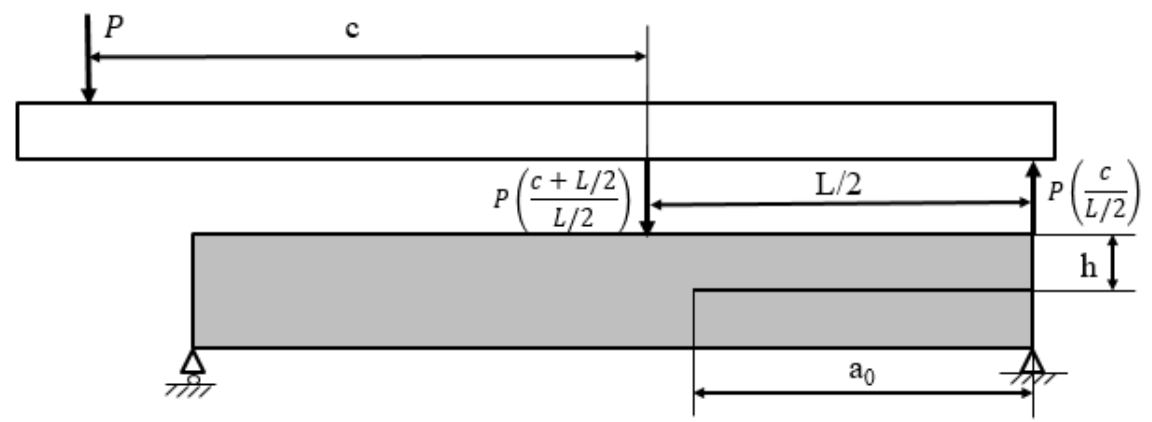

Figure 15: Mixed-mode bending specimen.

model. Each load-displacement drop corresponds to the global analysis recalculated with updated properties. The softening behavior of the load-displacement curve obtained with the global-local approach demonstrated good agreement with the analytical solution, whereas onset of delamination was predicted later than in the full 3D reference model and analytical results. The coupling results for the single-mode loadings are slightly more accurate than for the mixed-mode loading. However, after the first coupling step, the solution of the global-local model tended to the reference model curve resulting in less than $7 \%$ relative difference in the delamination initiation load $(143.8 \mathrm{kN}$ for the reference model and $153.5 \mathrm{kN}$ for the global-local model). In the Table 8 a comparison between the debonded areas of the local model and the corresponding region of the reference model is presented for each global-local coupling step. During the first coupling analysis, the cohesive elements in the local model were not completely damaged and, hence, were not deleted. However, due to the partial damage the energy was dissipated, which in turn led to the reduction of the global interlaminar stiffness and consequently a load drop. Nevertheless, after the second coupling step, the results of the reference and local model demonstrated a good agreement. 


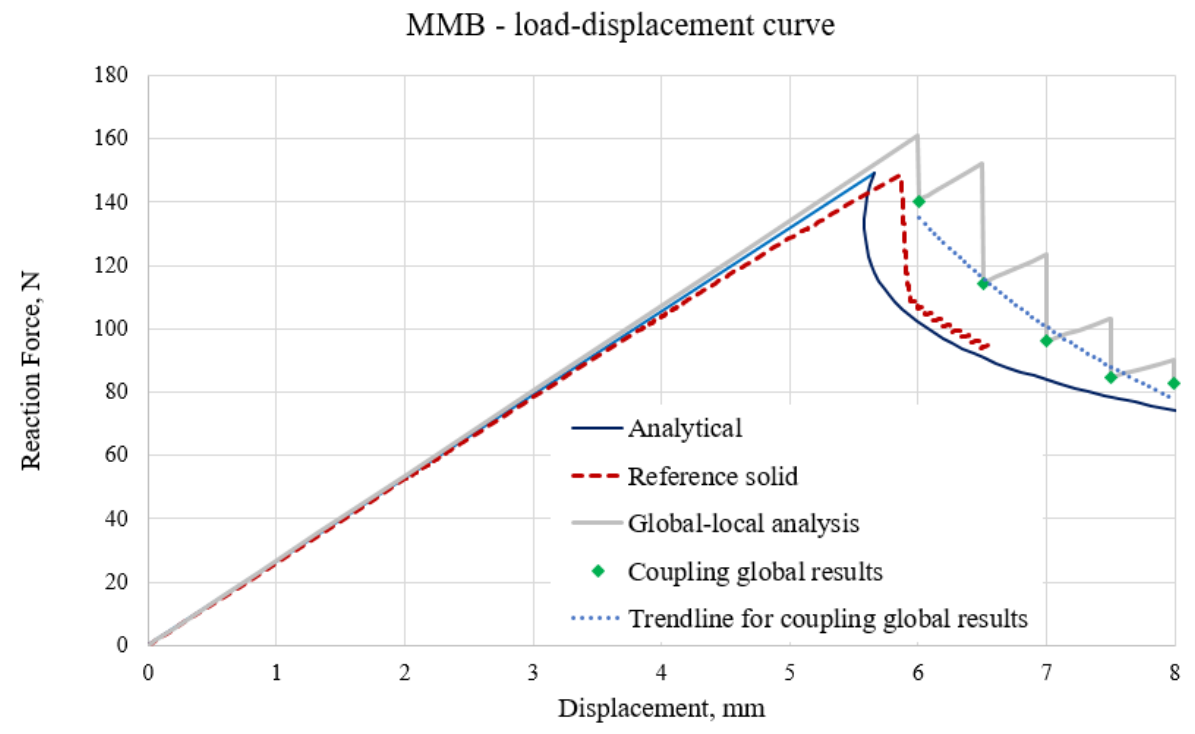

Figure 16: Load-displacement plot for the MMB specimen test.

Table 8: Comparison between debonded area of reference and local models.

\begin{tabular}{lrrrrr}
\hline Model & Step 1 & Step 2 & Step 3 & Step 4 & Step 5 \\
\hline Reference model, debonded area, $m m 2$ & 300 & 420 & 520 & 620 & 700 \\
Local model, debonded area, $m m 2$ & 0 & 380 & 500 & 560 & 600 \\
\hline
\end{tabular}

\subsection{Discussion}

The results of the pure and mixed-mode delamination cases presented herein show that the proposed global-local coupling approach can predict interlaminar damage in composite structures. Discrepancies between the coupling and reference analyses were observed, but these can be attributed to the coarse mesh employed in the global model, leading to the discrete load drops obtained by the coupling approach.

For instance, during the first coupling step, the global model indicates damage initiation slightly later due to the coarse mesh discretization. But it also leads to a sudden reduction of the stiffness, which in turn results in sudden load 
drops.

On the other hand, the reference model employs a fine mesh of solid and cohesive elements. To achieve convergence after delamination initiation, Abaqus implicit solver automatically reduces the increments within the step, leading to an almost progressive delamination propagation. In the global-local approach, the coarse mesh of the global model leads to a more abrupt extension of delamination at each displacement increment as a result of the stiffness degradation of the connector elements following the local analysis. These abrupt delamination extensions lead to the large load drops observed in the coupling results.

It is also important to emphasize that the updated degraded stiffness is applied in the global model only at the displacement level where the damage was predicted by the global model. This inevitably leads to the load drops in the global response prediction. Nevertheless, after the first coupling step, the results tend to the reference solution.

In addition, the effects of mesh and incrementation are expected to be reduced when applying the coupling approach to more complex models. This becomes clear in the analysis of the one-stringer stiffened panel (Section 44).

It should be noted that the main goal of the approach is to capture a nonlinear interlaminar material behaviour with a linear global model only correcting the global interlaminar stiffnesses after each global-local coupling step. Hence, the coupling steps should be coarse by nature. If the global response was not 520 corrected through the global-local procedure, the global solution would deviate more and more from the nonlinear reference solution with an increase of the applied displacement in the nonlinear regime of the load-displacement curve. Thus, the global response should be always interpreted as a coarse representation - stepwise damage propagation through a finite number of coupling steps 525 - of the actual response of the structure. To conclude, the progressive damage extension modelled by means of the global-local technique gives a robust prediction of damage propagation at a reduced computational cost. 
Table 9: Geometry of stiffened composite panel.

\begin{tabular}{ll}
\hline Description & Value \\
\hline Panel length, $l(\mathrm{~mm})$ & 100 \\
Panel width, $w(\mathrm{~mm})$ & 40 \\
Stringer width, $b(\mathrm{~mm})$ & 20 \\
Stringer height, $h(\mathrm{~mm})$ & 8 \\
Laminate thickness, $t_{\text {skin }}, t_{\text {blade }}(\mathrm{mm})$ & 1 \\
Adhesive thickness, $t_{\text {adh }}(\mathrm{mm})$ & 0.2 \\
\hline
\end{tabular}

\section{One-stringer stiffened panel under compression}

In order to investigate skin-stringer debonding the developed approach has been implemented to a stiffened composite panel with one T-stringer loaded under compression, see Fig. 17. This panel has been already examined through the global-local approach with the averaging procedure of degraded parameters, see [29]. The new method that ensures transition of dissipated energy from local to the global level, and thus accounting for local effects in the global model, has been used for the current analysis. The skin and the stringer of the panel is composed of unidirectional symmetrical layups $[0,90]_{s}$. The axial compressive load is applied to the transverse edges, whereas the other edge is clamped on all directions, except for the longitudinal. Material and geometry parameters are summarized in Tables 9 and 10 respectively.

\subsection{Global model: linear elasticity}

The global model was created with conventional 4-node shell elements with reduced integration ( $\mathrm{S} 4 \mathrm{R}$ in Abaqus) and $5 \mathrm{~mm}$ size. Buckling was triggered by an imperfection set as a first eigenmode of linear analysis. The ${ }^{*}$ Offset parameter was applied to move the reference shell surfaces of the stringer and the skin from the middle surfaces towards the lower and upper surfaces respectively, so that connector elements will have real lengths of the interface thickness, see 


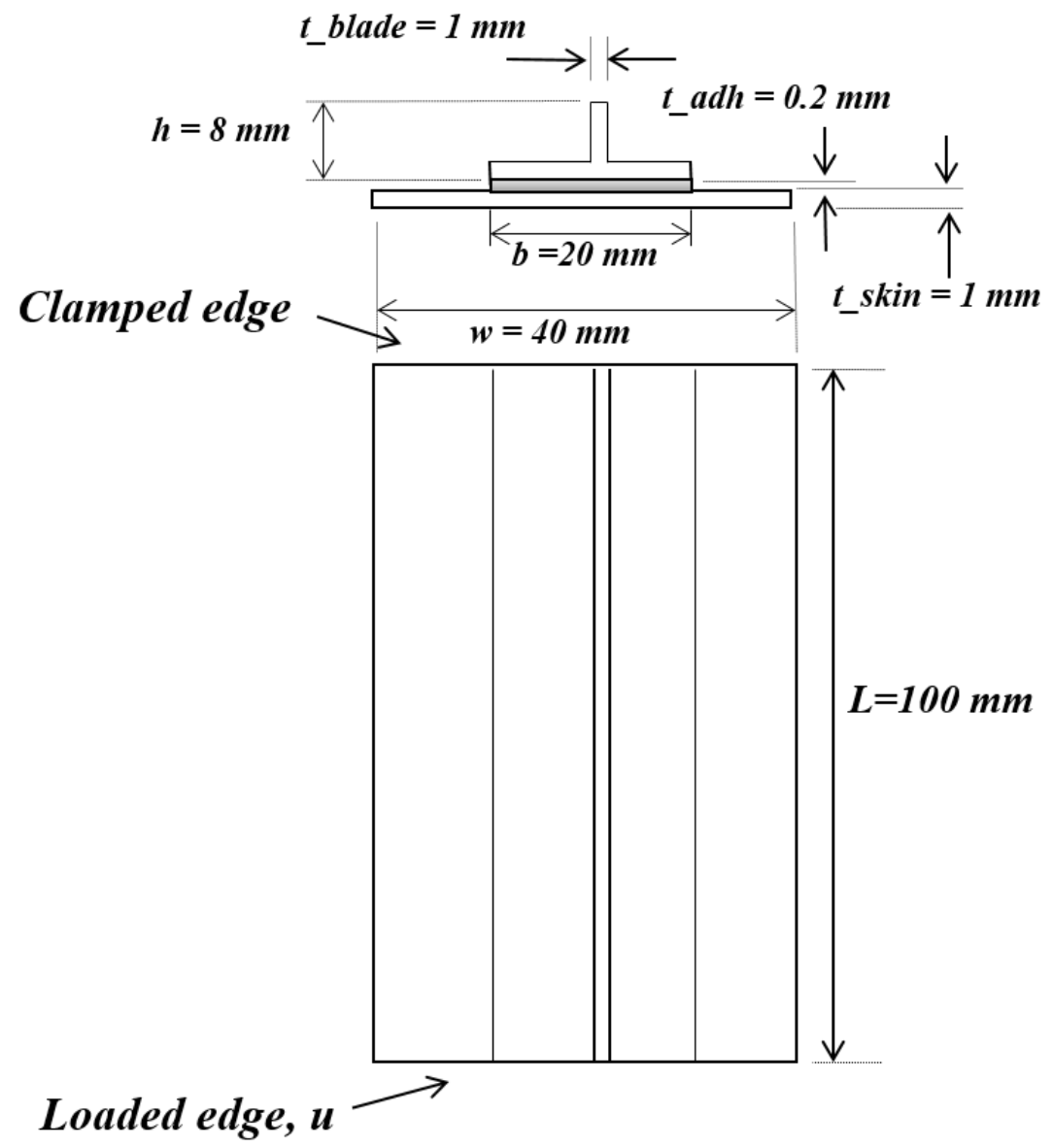

Figure 17: Geometry of stiffened panel.

Fig. 18. The quadratic stress criterion from Eq. 3 is used to detect the critical areas of the skin-stringer debonding initiation. During the consecutive coupling loops, the global stiffnesses of the connector elements were degraded following the procedure described in detail for the mixed-mode loading case.

\subsection{Local models: nonlinear material model}

Local models were built with linear solid elements (C3D8 in Abaqus) of $1 \mathrm{~mm}$ length used to model the skin and the stringer and non-zero thickness cohesive elements (COH3D8 in Abaqus) used for the interface layer to examine 
Table 10: Material data for composite and adhesive.

\begin{tabular}{ll}
\hline Stiffness properties & Value \\
\hline Young's modulus in 1-direction, $E_{11}(\mathrm{GPa})$ & 146.5 \\
Young's modulus in 2-direction, $E_{22}(\mathrm{GPa})$ & 9.7 \\
Shear modulus in 12-plane, $G_{12}(\mathrm{GPa})$ & 5.1 \\
Poisson's ratio, $\nu_{12}$ & 0.28 \\
Young's modulus of adhesive, $E_{\text {glue }}(\mathrm{GPa})$ & 3.0 \\
Poisson's ratio of adhesive $\nu_{\text {glue }}$ & 0.4 \\
\hline
\end{tabular}

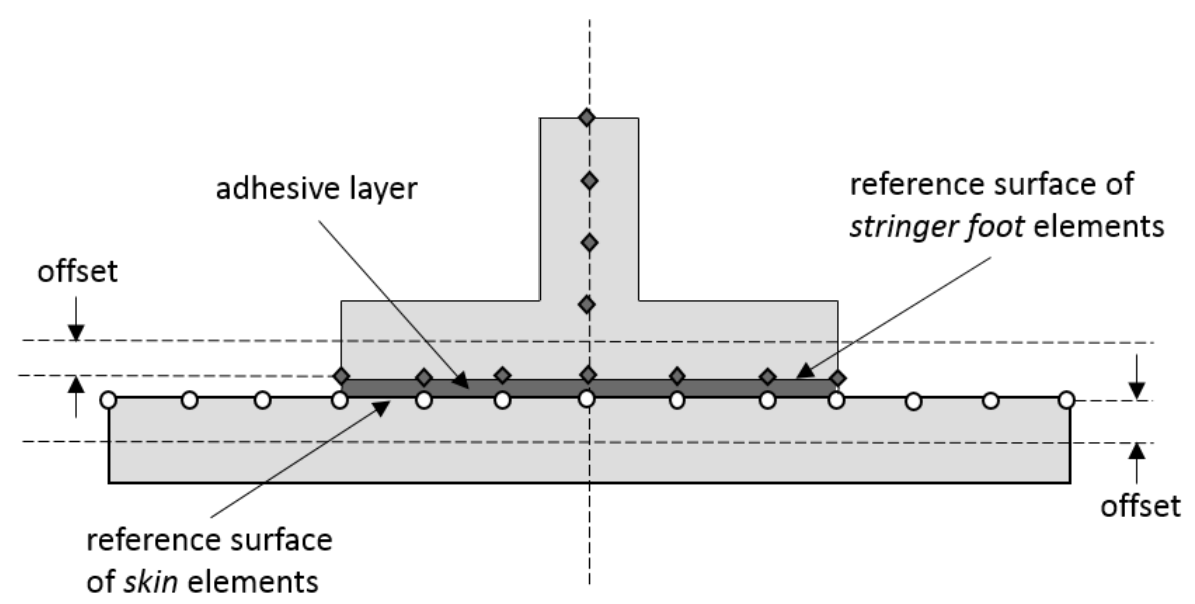

Figure 18: Geometry of a section of the stiffened panel.

555

skin-stringer debonding onset and growth. It is worth mentioning that application of higher order solid elements was not leading to improvements in damage prediction. One solid element per lamina in the thickness direction was used. Following the suggestion in [29], relatively high viscosity of $10^{-3}$ and four cohesive elements per solid element were selected, resulting in $0.25 \mathrm{~mm}$ in-plane size. Their strength and fracture toughness are listed in Table 11.

It is important to mention that the local models are generated based on the size and location of the damage identified during the global analysis by using 
Table 11: Material data for cohesive elements.

\begin{tabular}{ll}
\hline Cohesive element properties & Value \\
\hline Interfacial strength, mode I, $\tau_{I}(\mathrm{MPa})$ & 61 \\
Interfacial strength, mode II and III, $\tau_{I I}, \tau_{I I I}(\mathrm{MPa})$ & 68 \\
Fracture toughness, mode I, $G_{I c}(\mathrm{~N} / \mathrm{mm})$ & 0.243 \\
Fracture toughness, mode II and III, $G_{I I c}, G_{I I I c}(\mathrm{~N} / \mathrm{mm})$ & 0.514 \\
\hline
\end{tabular}

a MATLAB preprocessor. The output parameters of the script are the nodes and elements for the local models. Thus, when the delamination propagates the local models are increased to include the expansion of the damaged area.

After completion of the local analysis calculations, the degraded stiffness of each global connector element was updated based on the approach described in section 2 for the mixed-mode loading case.

\subsection{Reference solid model}

The reference solid model consisted of linear solid elements and cohesive elements with the same mesh density chosen for the local model. An initial geometrical imperfection to trigger buckling was included using the first eigenmode as for the global model.

\subsection{Coupling results}

Six global-local coupling steps were performed similarly to the work in 29 ] with the following increase of the applied displacement: $0.56 \mathrm{~mm}, 0.58 \mathrm{~mm}$, $0.60 \mathrm{~mm}, 0.63 \mathrm{~mm}, 0.67 \mathrm{~mm}$, and $0.82 \mathrm{~mm}$. The convergence in reaction forces has been attained after several iterations for each coupling step, followed by an increase of the prescribed displacement. Local models based on the critical regions and determined at the global level have been created. Overlay plots for the first, third and fourth coupling steps are demonstrated in Fig. 19 as the most representative for the current examination. Two local models corresponded to 


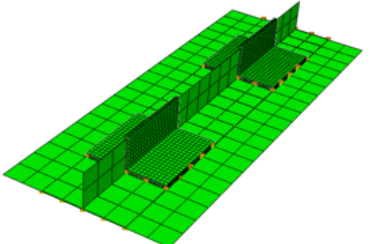

Step 1: $u=0.56 \mathrm{~mm}$

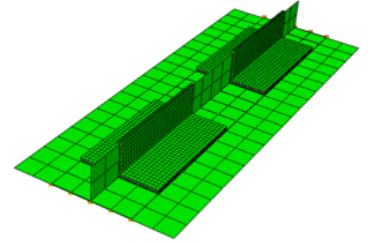

Step 3: $u=0.60 \mathrm{~mm}$

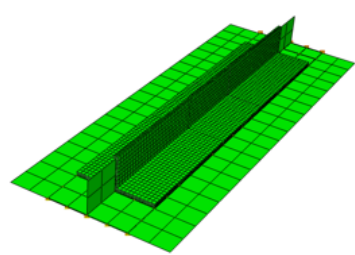

Step 4: $u=0.63 \mathrm{~mm}$

Figure 19: Overlay plots of the global and local models of the stiffened panel for coupling steps 1,3 and 4 .

the first two coupling steps. They were placed symmetrically each covering an area of $400 \mathrm{~mm}^{2}$ of the adhesive surface during the first and the second coupling steps. The size and the location of these models were unchanged for these first two steps, as the increased area of damaged connector elements were fully covered by these models. Expansion of the skin-stringer debonding initiation detected at the global level provoked an enlargement of the local models to $600 \mathrm{~mm}^{2}$ of adhesive surface covered during the third coupling step.

It is important to mention that the onset of the debonding started at the free edge surfaces between the skin and the stringer and propagated in the direction of the web of the stringer. During the fourth coupling step the third local model was generated according to a new set of critical connector elements. It should be noted that although three local models could be joined in one large local model, this approach was regarded as undesirable. Separate local models allowed for the parallel computation and proved to be more computationally effective without much loss in accuracy. During the fifth and sixth coupling steps the same local models were employed as they fully covered the damaged areas.

Fig. 20 shows the load-displacement curves for the global-local coupling analyses based on dissipated energies and based on averaging of damage variables [29] and full 3D reference solution. The difference in the first buckling displacement between global-local approaches $(0.147 \mathrm{~mm})$ and the reference solution 


\section{Load-displacement curve}

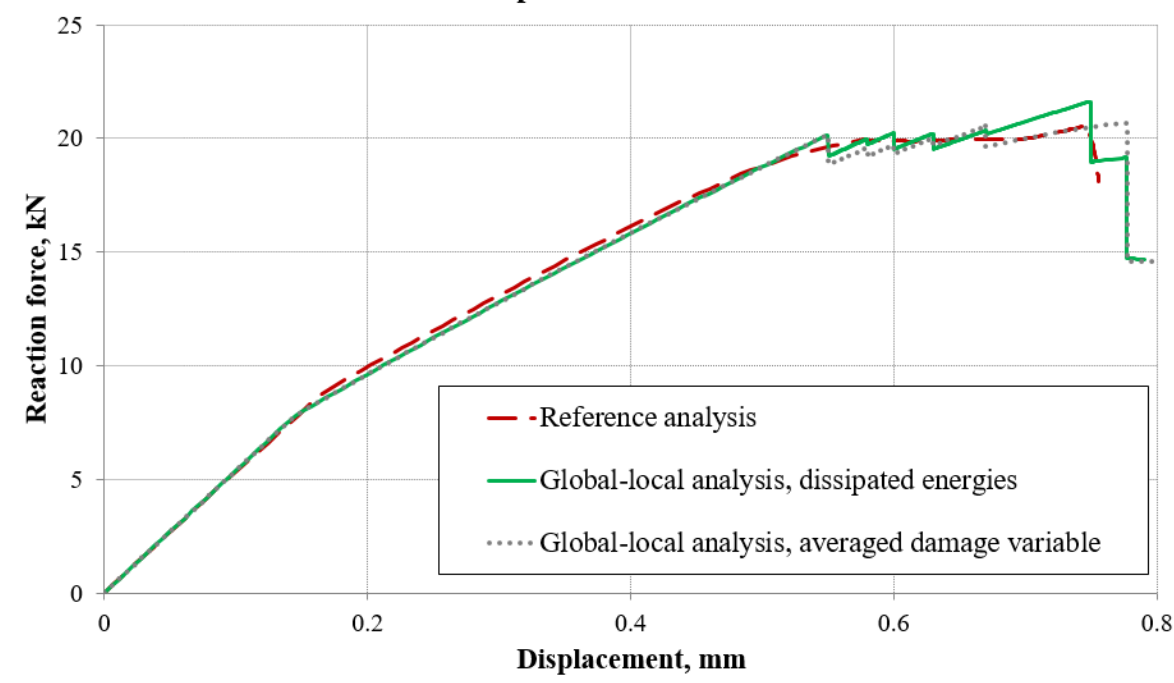

Figure 20: Load-displacement curves for progressive failure analysis of the stiffened panel. Two approaches: global-local method based on dissipated energies and global-local method based on averaging of damage parameter.

$(0.167 \mathrm{~mm})$ leads to the slight difference in the curves, although the structural

stiffness was predicted very well. In the postbuckling regime the global-local curves also match well with the reference curve by virtue of the slight drops of the global-local curves that adjust the overall global behavior after each globallocal coupling step. The final collapse of the structure is defined by a large drop in the load-displacement curve which implies dramatic reduction in the load-carrying capacity. The final failure in the global-local simulation based on dissipated energies occurs at a load level of $22.35 \mathrm{kN}$ which corresponds to the displacement of around $0.75 \mathrm{~mm}$. The final collapse predicted by the global-local model based on the assumption of the averaged damage variables occurred at a load level of $20.68 \mathrm{kN}$ and a displacement of $0.78 \mathrm{~mm}$. The maximum carried load by the reference model is $20.54 \mathrm{kN}$ at the displacement of $0.76 \mathrm{~mm}$. At around $0.77 \mathrm{~mm}$ of applied displacement the global buckling shape changed and the stringer kinked leading to the drop in the load-displacement curve of the global-local analysis. The comparison with the reference model demonstrated 
that due to incremental damage the complete failure of the reference model

took place slightly earlier at $0.76 \mathrm{~mm}$, which did not allow to capture the same effect. However, it should be noted that the displacement of the final collapse, corresponding to a sudden drop of the load-displacement curve, of the reference analysis was predicted very closely by the global-local method based on the dissipated energies, whereas the maximum supported load was slightly overestimated resulting in $9 \%$ of relative difference. It should be noted that only delamination growth is considered in all models.

A comparison between the two global-local approaches has been conducted in terms of the dissipated energies. The results are demonstrated in Table 12 The local model selected, denoted "Local model 1", corresponds to the initial damaged area located symmetrically with respect to the stringer and grows with increasing damage. The dissipated energy in the reference model was calculated for the region corresponding to the region of the local model. Although both results for the local models show discrepancies with respect to the reference model, it is noted that a difference in the prediction of the damage initiation will take place due to the difference in mesh refinement. In the reference model the criterion, which identifies the damage initiation, is checked iteratively. In the global model the same happens but during the post-processing procedure, hence, with lower accuracy, as the global model has a coarser mesh that consists of shell elements. Nevertheless, the dissipated energy in the local model of the averaging global-local approach presented in [29] clearly shows a considerably larger difference as compared with the reference model results. In the averaging procedure in the method presented in 29] (also resulting in reasonable results for the global-local progressive failure analysis) the equivalence between the dissipated energies of the global and local models was not enforced.

A comparison of the mix-mode ratios at damage initiation in the cohesive elements has been performed for the reference model, for the local model based on the averaging procedure and for the local model obtained following the preservation of dissipated energies, illustrated in Fig. 21. This demonstrates that, although both local models of different approaches give similar results to the 
Table 12: Energy dissipated in the local and reference models of the stiffened panel due to the skin-stringer separation for 6 coupling steps.

\section{Dissipated energy, $N \cdot m m$}

\begin{tabular}{lccc}
\hline Coupling step & $\begin{array}{c}\text { Local model 1 } \\
\text { Averaging }\end{array}$ & $\begin{array}{c}\text { Local model 1 } \\
\text { Diss. Energies }\end{array}$ & Reference model \\
\hline Coupling step 1, u=0.56 mm & 60.9 & 60.9 & 32.2 \\
Coupling step 2, $\mathrm{u}=0.58 \mathrm{~mm}$ & 167.4 & 80.2 & 50.2 \\
Coupling step 3, $\mathrm{u}=0.60 \mathrm{~mm}$ & 235.0 & 185.6 & 80.6 \\
Coupling step 4, $\mathrm{u}=0.63 \mathrm{~mm}$ & 388.0 & 228.8 & 122.2 \\
Coupling step 5, $\mathrm{u}=0.67 \mathrm{~mm}$ & 435.5 & 263.0 & 182.6 \\
Coupling step 6, $\mathrm{u}=0.82 \mathrm{~mm}$ & 604.9 & 303.8 & 330.9 \\
\hline
\end{tabular}

Table 13: Comparison of relative difference of mixed-mode ratio of damage initiation in reference model at the displacement of $0.58 \mathrm{~mm}$ and local models 1 obtained from the averaging procedure and for the preservation of the energies dissipated after the Coupling step 2 (corresponding to a displacement of $0.58 \mathrm{~mm}$ at the global level).

\begin{tabular}{ccc}
\hline \multicolumn{3}{c}{ Percentage of cohesive elements, \% } \\
\hline $\begin{array}{c}\text { Relative difference } \\
\text { with ref. model }\end{array}$ & $\begin{array}{c}\text { Local model 1 } \\
\text { Averaging }\end{array}$ & $\begin{array}{c}\text { Local model 1 } \\
\text { Diss. Energies }\end{array}$ \\
\hline$\leq 10 \%$ & 68.8 & 75.4 \\
$\leq 15 \%$ & 73.2 & 79.4 \\
$\leq 20 \%$ & 74.4 & 80.5 \\
\hline
\end{tabular}




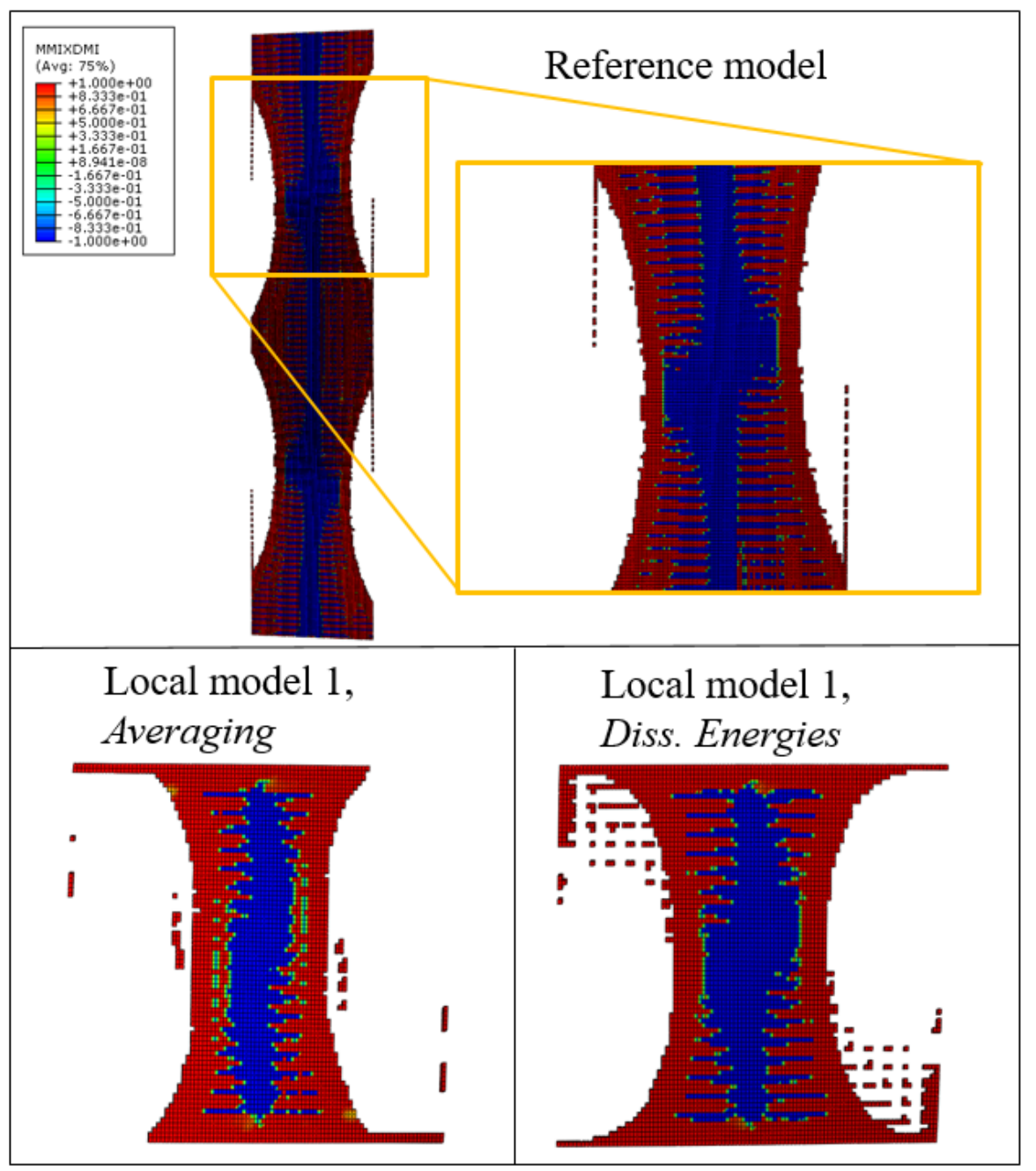

Figure 21: Mixed-mode ratio of the damage initiation for cohesive elements of the reference model at the displacement of $0.58 \mathrm{~mm}$ and local models 1 obtained from the averaging procedure and for the preservation of the energies dissipated after the Coupling step 2 (corresponding to a displacement of $0.58 \mathrm{~mm}$ at the global level).

reference solution, the way the damage propagates in terms of the correct damage mode is predicted closer by the method that preserves dissipated energies across the levels. Table 13 shows that percentage of cohesive elements in the local model 1 obtained by means of the dissipated energy preservation technique 
is higher as compared to the same local model from the averaging procedure. For instance, $75.4 \%$ of cohesive elements from the energy-based method lie within $10 \%$ relative difference to reference model cohesive elements when the mixed-mode ratios of damage initiation are compared, whereas for the averaging approach this value is $68.8 \%$. Hence, on the one hand, an application of the new method to a one-stringer panel confirmed that the method attains good agreement with the reference calculations. On the other hand, it also showed that for the chosen example the final collapse as well as skin-stringer separation are predicted well by both approaches with a difference that is explained in the following. The global-local coupling method which is based on the preservation of the dissipated energies between the local and global levels, leads to a closer prediction of the mixed-mode damage initiation in comparison to the reference solution. However, it does not have large influence on the load-displacement curve as well as on the locations of the damaged areas. As the debonding started at the free edges due to the excessive buckling in this case, it was not so important how exactly the adhesive properties were reduced as it did not affect the path of the damage extension. But it is important to emphasize that for larger models with multiple delaminations this accuracy in predicting the damage modes could have an impact on the damage redistribution.

To conclude, it is important to notice that the global-local approach based on the preservation of the energy dissipation demonstrated its effectiveness and accuracy in application to a complex structure.

\section{Conclusion}

A novel two-way coupling global-local finite element approach for delamination preserving dissipated energy at both global and local levels has been formulated. This method establishes a procedure of dissipated energy calculation for the global model due to delamination based on the dissipated energy in the local model. This new global-local method also permits a reliable and efficient simulation of the delamination propagation by virtue of information 
exchange between separated global and local models. Single-mode and mixedmode loading cases were examined for the first time as benchmark applications of the proposed methodology, showing that the approach captures the physics of delamination propagation correctly. In particular, the double cantilever beam (DCB) test, the end notched flexure (ENF) test and the mixed-mode loading (MMB) test were considered. In regard to the DCB benchmark, the results obtained for the global-local coupling approach correlate with a good agreement to the analytical and reference numerical results. In the case of the ENF test, the global-local approach also showed the ability to predict the delamination response closely to the analytical solution. With respect to the MMB test, the global-local numerical solution was able to capture the softening part of the loaddisplacement curve, but delamination initiation was predicted later than in the analytical solution and, thus, the overall structural strength was overestimated. This is due to the fact that accurate prediction of the delamination onset with a coarse mesh is not possible and global models provide an estimation of critical areas, whereas local models with finer mesh are utilized for detailed analysis. The skin-stringer debonding onset and propagation leading to final collapse was examined with this global-local approach as a further validation, resulting in an excellent agreement with a full 3D reference numerical simulation proving the robustness of the method. The developed global-local numerical method is an effective tool for modeling delamination propagation at separated global and local levels, ensuring that the energy dissipated due to delamination evolution 705 at the local level will be captured at the global level, triggering the reduction of load-bearing capacity at the global level up to the final collapse. In the future, the proposed strategy could be applied to more complex scenarios (e.g. impact loading of stiffened panels) structures to exploit the potential of the global-local method. 


\section{Acknowledgments}

The research leading to these results has received funding from European Union's Horizon 2020 research and innovation program (FULLCOMP/20152019) under Marie Sklodowska-Curie actions grant agreement number 642121. The provided financial support is gratefully acknowledged by the authors.

[1] R. Degenhardt, A. Kling, H. Klein, W. Hillger, H. C. Goetting, R. Zimmermann, K. Rohwer, Experiments on buckling and postbuckling of thinwalled cfrp structures using advanced measurement systems, International Journal of Structural Stability and Dynamics 7 (2) (2007) 337-358.

[2] S. Hühne, J. Reinoso, E. Jansen, R. Rolfes, A two-way loose coupling procedure for investigating the buckling and damage behaviour of stiffened composite panels, Composite Structures (136) (2016) 513-525. doi: $10.1016 / \mathrm{j}$.compstruct.2015.09.056.

[3] M. Akterskaia, E. Jansen, S. Hühne, R. Rolfes, Efficient progressive failure analysis of multi-stringer stiffened composite panels through a two-way loose coupling global-local approach, Composite Structures 183 (2018) 137145. doi:10.1016/j.compstruct.2017.02.011.

[4] M. Akterskaia, E. Jansen, S. Hallett, P. Weaver, R. Rolfes, Analysis of skin-stringer debonding in composite panels through a two-way global-local method, Composite Structures doi:10.1016/j . compstruct.2018.06.064

[5] R. Krueger, The virtual crack closure technique for modeling interlaminar failure and delamination in advanced composite materials, Elsevier Ltd., 2015. doi:10.1016/B978-0-08-100332-9.00001-3.

[6] E. Rybicki, M.F. Kanninen, A finite element calculation of stress intensity factors by modified crack closure integral, Engineering Fracture Mechanics 9 (1977) 931-938. doi:doi.org/10.1016/0013-7944(77)90013-3.

[7] M. Crisfield, Progressive delamination using interface elements (1998). 
[8] G. Alfano, M. a. Crisfield, Finite element interface models for the delamination analysis of laminated composites: \mboxmechanical and computational issues, Int. J. Numer. Meth. Eng. 50 (March 2000) (2001) 1701-1736.

[9] P. Camanho, C. Davila, Mixed-mode decohesion finite elements in for the simulation composite of delamination materials, Nasa TM-2002-21 (June) (2002) 1-37. doi:10.1177/002199803034505

[10] D. S. Dugdale, Yielding of steel sheets containing slits, J. Mech. Phys. Solids 8 (1960) 100-104.

[11] G. I. Barenblatt, The mathematical theory of equilibrium cracks in brittle fracture, Advances in Applied Mechanics 7 (C) (1962) 55-129. doi:10. 1016/S0065-2156(08)70121-2.

[12] A. Hillerborg, M. Modeer, P.-E. Petersson, Analysis of crack formation and crack growth in concrete by means of fracture mechanics and finite elements, Cement and concrete research 6 (1976) 773-782.

[13] O. Allix, P. Ladevèze, Interlaminar interface modelling for the prediction of laminate delamination, Composite Structures 22 (1992) 235-242.

[14] A. Turon, P. P. Camanho, A damage model for the simulation of delamination in advanced composites under variable-mode loading, Mechanics of Materials 38 (2006) 1072-1089. doi:10.1016/j.mechmat.2005.10.003

[15] R. Borg, L. Nilsson, K. Simonsson, Simulation of delamination in fiber composites with a discrete cohesive failure model, Composites Science

口 and Technology 61 (5) (2001) 667-677. doi:10.1016/S0266-3538(00) 00245-1.

[16] M. R. Wisnom, F. K. Chang, Modelling of splitting and delamination in notched cross-ply laminates, Composites Science and Technology 60 (15) (2000) 2849-2856. doi:10.1016/S0266-3538(00)00170-6 
[17] D. Xie, A. M. Waas, Discrete cohesive zone model for mixed-mode fracture using finite element analysis, Engineering Fracture Mechanics 73 (13) (2006) 1783-1796. doi:10.1016/j.engfracmech.2006.03.006

[18] S. R. Hallett, M. R. Wisnom, Numerical investigation of progressive damage and the effect of layup in notched tensile tests, Journal of Composite Materials 40 (14) (2006) 1229-1245. doi:10.1177/0021998305057432.

[19] W.-G. Jiang, S. R. Hallett, B. G. Green, M. R. Wisnom, A concise interface constitutive law for analysis of delamination and splitting in composite materials and its application to scaled notched tensile specimens, International Journal for Numerical Methods in Engineering 69 (9) (2007) 1982-1995. doi:10.1002/nme.1842

[20] X. Liu, R. Duddu, H. Waisman, Discrete damage zone model for fracture initiation and propagation, Engineering Fracture Mechanics 92 (2012) 1-18. doi:10.1016/j.engfracmech.2012.04.019

[21] S. Jimenez, X. Liu, R. Duddu, H. Waisman, A discrete damage zone model for mixed-mode delamination of composites under high-cycle fatigue, International Journal of Fracture 190 (2014) 53-74. doi:10.1007/ s10704-014-9974-0.

[22] M. Cabello, A. Turon, J. Zurbitu, J. Renart, C. Sarrado, F. Martínez, Progressive failure analysis of dcb bonded joints using a new elastic foundation coupled with a cohesive damage model, European Journal of Mechanics, A/Solids 63 (2017) 22-35. doi:10.1016/j.euromechsol.2016.12.004.

[23] A. C. Orifici, I. O. de Zarate Alberdi, R. S. Thomson, J. Bayandor, Compression and post-buckling damage growth and collapse analysis of flat composite stiffened panels, Composites Science and Technology 68 (15-16) (2008) 3150-3160. doi:10.1016/j.compscitech.2008.07.017.

[24] J. Reinoso, A. Blázquez, A. Estefani, F. París, J. Cañas, E. Arévalo, F. Cruz, Experimental and three-dimensional global-local finite element 
analysis of a composite component including degradation process at the compositesb.2012.02.010.

[25] R. Vescovini, C. G. Dávila, C. Bisagni, Failure analysis of composite multistringer panels using simplified models, Composites Part B 45 (1) (2013) 939-951. doi:10.1016/j.compositesb.2012.07.030.

[26] R. Borrelli, A. Riccio, A. Sellitto, F. Caputo, T. Ludwig, On the use of global local kinematic coupling approaches for delamination growth simulation in stiffened composite panels, Composites Scienece and Technology 115 (2015) 43-51. doi:10.1016/j.compscitech.2015.04.010.

[27] O. Bettinotti, O. Allix, U. Perego, V. Oancea, B. Malherbe, Simulation of delamination under impact using a globallocal method in explicit dynamics, Finite Elements in Analysis and Design 125 (June 2016) (2017) 1-13. doi: 10.1016/j.finel.2016.11.002.

[28] K. Saavedra, O. Allix, P. Gosselet, J. Hinojosa, A. Viard, An enhanced nonlinear multi-scale strategy for the simulation of buckling and delamination on 3d composite plates, Computer Methods in Applied Mechanics and Engineering 317 (2017) 952-969. doi:10.1016/j.cma.2017.01.015.

[29] M. Akterskaia, E. Jansen, R. Rolfes, Progressive failure analysis of stiffened composite panels using a two-way loose coupling approach including intralaminar failure and debonding, 2018 AIAA/ASCE/AHS/ASC Structures, Structural Dynamics, and Materials Conference (2018) 1-16doi: $10.2514 / 6.2018-0735$

[30] J. R. Reeder, J. H. Crews, Mixed-mode bending method for delamination testing, AIAA Journal 28 (7) (1990) 1270-1276. doi:10.2514/3.25204.

[31] M. L. Benzeggagh, M. Kenane, Measurement of mixed-mode delamination fracture toughness of unidirectional glass / epoxy composites with mixed- 
mode bending apparatus, Composites Science and Technology 56 (1996) $439-449$.

${ }_{820}$ [32] A. Turon, E. V. González, C. Sarrado, G. Guillamet, P. Maimí, Accurate simulation of delamination under mixed-mode loading using a cohesive model with a mode-dependent penalty stiffness, Composite Structures

(1) 184 (September 2017) (2018) 506-511. doi:10.1016/j.compstruct. 2017. 10.017 .

[33] A. Turon, P. P. Camanho, J. Costa, J. Renart, Accurate simulation of delamination growth under mixed-mode loading using cohesive elements: Definition of interlaminar strengths and elastic stiffness, Composite Structures 92 (8) (2010) 1857-1864. doi:10.1016/j.compstruct.2010.01.012

[34] E. V. Gonzalez, P. Maimi, A. Turon, P. P. Camanho, J. Renart, Simula830 tion of delamination by means of cohesive elements using an explicit finite element code, Cmc-Computers Materials \& Continua 9 (1) (2009) 51-92. doi:10.3970/cmc.2009.009.051. 